ARTICLE

\title{
Structural insights into assembly of the ribosomal nascent polypeptide exit tunnel
}

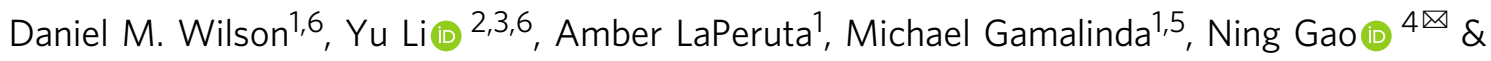
John L. Woolford Jr (D) ${ }^{1 凶}$

The nascent polypeptide exit tunnel (NPET) is a major functional center of 605 ribosomal subunits. However, little is known about how the NPET is constructed during ribosome assembly. We utilized molecular genetics, biochemistry, and cryo-electron microscopy (cryoEM) to investigate the functions of two NPET-associated proteins, ribosomal protein uL4 and assembly factor Nog1, in NPET assembly. Structures of mutant pre-ribosomes lacking the tunnel domain of uL4 reveal a misassembled NPET, including an aberrantly flexible ribosomal RNA helix 74, resulting in at least three different blocks in 60 S assembly. Structures of preribosomes lacking the C-terminal extension of Nog1 demonstrate that this extension scaffolds the tunnel domain of $\mathrm{uL} 4$ in the NPET to help maintain stability in the core of pre-60S subunits. Our data reveal that uL4 and Nog1 work together in the maturation of ribosomal RNA helix 74, which is required to ensure proper construction of the NPET and 60S ribosomal subunits.

\footnotetext{
${ }^{1}$ Department of Biological Sciences, Carnegie Mellon University, Pittsburgh, PA 15213, USA. ${ }^{2}$ State Key Laboratory of Membrane Biology, School of Life Science, Tsinghua University, Beijing, China. ${ }^{3}$ Peking University-Tsinghua University-National Institute of Biological Sciences Joint Graduate Program, Beijing, China. ${ }^{4}$ State Key Laboratory of Membrane Biology, Peking-Tsinghua Center for Life Sciences, School of Life Sciences, Peking University, Beijing, China. ${ }^{5}$ Present address: Bayer AG, Strategy and Business Consulting, Building B151, 51368 Leverkusen, Germany. ${ }^{6}$ These authors contributed equally: Daniel M. Wilson, Yu Li.凶email: gaon@pku.edu.cn; jw17@andrew.cmu.edu
} 
T ranslation of the genetic code and catalysis of peptide bond formation to synthesize proteins is carried out by ribosomes. Assembly of ribosomes requires the transcription, folding, modification, and processing of ribosomal RNA (rRNA), and stable association of ribosomal proteins (RPs) facilitated by trans-acting assembly factors (AFs). In eukaryotes, this process takes place in three separate subcellular compartments beginning in the nucleolus, continuing in the nucleoplasm, and concluding in the cytoplasm. To ensure their proper function, these complex ribonucleoprotein particles must undergo accurate assembly of their functional centers including the decoding center (DC) in the small subunit (40S) and the peptidyltransferase center (PTC) and nascent polypeptide exit tunnel (NPET) in the large subunit $(60 S)^{1,2}$.

The NPET is the 90 angstrom-long conduit through which all nascent polypeptide chains travel from the PTC to the exterior of the $60 \mathrm{~S}$ subunit ${ }^{3}$. It has become increasingly clear that the NPET functions as a sensor of both specific sequences in nascent polypeptides and small molecule effectors, including antibiotics, to modulate the activity of the PTC and regulate the synthesis of specific subsets of proteins ${ }^{4-6}$. The physicochemical properties of the NPET have also been shown to actively affect protein folding $^{7}$. Thus, we are particularly interested to learn how cells ensure a properly constructed NPET.

The crystal structure of mature yeast ribosomes first revealed that the eukaryotic NPET is composed of rRNA from five out of six domains of $25 \mathrm{~S}$ rRNA, and the RPs uL4 (rpL4), uL22 (rpL17), and eL39 $(\mathrm{rpL39})^{8}$. Some of the most conserved elements are the constriction sites formed by the internal loops of uL4 and uL22. In eukaryotes, the bilobal tunnel domain (TD) at the tip of the uL4 internal loop forms two constriction sites as opposed to one found in bacteria (Fig. 1a) ${ }^{9}$. The internal loops of both uL4 and uL22 have each been shown to be important for the assembly and function of ribosomes in both prokaryotes and eukaryotes ${ }^{10-14}$. Additionally, eL39 further distinguishes eukaryotic from prokaryotic NPETs and can help explain differences between translation modes observed between the two .

Recent cryo-electron microscopy (cryo-EM) of native yeast pre-60S subunits has provided numerous structural snapshots of assembly intermediates, including glimpses of ordered stages of NPET construction (Fig. 1a, b). In early nucleolar pre-60S particles (Nsal state 2), a precursor to the tunnel is visible, with the long alpha helix of AF Rrp14 sterically hindering incorporation of $25 \mathrm{~S}$ rRNA domain II into the NPET (Fig. 1a) ${ }^{15}$. At this stage, $\mathrm{uL} 4$ is present in preribosomes but the TD of $\mathrm{UL} 4$ is not visible and thus is likely to be in a flexible state. Next, in Nsal state C particles, Rrp14 has been released, the aminoterminal domain of AF Rpf1 contacts portions of 25S rRNA domain I that will become part of the tunnel, and the uL4 TD has stabilized and becomes visible. A more complete tunnel-like structure forms in later nucleolar intermediates. In Nsal state E particles, Rpf1 has been released and the CTD of AF Spb1 traverses the upper portion of the tunnel, contacting the TD of uL4 at the first constriction site, possibly functioning to further stabilize uL4 in the NPET ${ }^{16}$. As pre-60S particles transition from the nucleolus to the nucleoplasm, several AFs are released and others join pre-60S subunits. In the ensuing Nog2 state 1 particles, the CTD of AF Nog1 can be seen occupying almost the full length of the tunnel, contacting the uL4 TD ${ }^{17}$, and eL39 is recruited to the tunnel exit. Upon release of Spb1, insertion of the Nog1 CTD may serve to maintain stability of the UL4 TD. Once preribosomes are exported to the cytoplasm (Arx1 particles), Nog1 is released by the AAA-ATPase Drg1 18,19 and replaced in the NPET by the CTD of AF Reil. Reil is then removed and replaced in the NPET by its homologue Reh1 (Nmd3 particles), which is released upon completion of $60 \mathrm{~S}$ subunit assembly ${ }^{20-22}$. The CTDs of both Rei1 and Reh1 also contact the TD of uL4. Thus, remarkably, the NPET is sequentially occupied by carboxy or amino-terminal extensions of AFs from early nucleolar stages of $60 \mathrm{~S}$ subunit assembly until final steps in the cytoplasm.

Despite this wealth of structural information, it remains unclear precisely how the NPET is constructed, what are the specific roles of ribosomal proteins and assembly factors in NPET assembly, and how NPET formation fits into the hierarchy of 60S subunit biogenesis. To investigate these questions, we focused on uL4 and Nog1, which contact each other inside the NPET during late nucleolar and nucleoplasmic (middle) stages of assembly (Fig. 1).

We used biochemical and molecular genetic analyses, together with cryo-EM, to investigate the effects on ribosome assembly when we deleted the TD of UL4 or the Nog1 CTD. We found that pre-60S particles lacking the TD of uL4 harbor a misassembled NPET that lacked the densities of eL39 and the Nog1 CTD and are blocked or stalled in at least three different stages of 60S subunit assembly. These blocks begin with rRNA helix H74 (H74), which lies in close proximity to the TD of uL4, being improperly assembled. Consequently, adjacent helices $\mathrm{H} 75$, H76, and $\mathrm{H} 68$, are also displaced. We also found that the Nog1 CTD functions to stabilize the UL4 TD and ensure proper maturation of H74. Thus, truncation of the Nog1 CTD causes an assembly defect similar, but not identical to that of deleting the uL4 TD. Our work suggests that the quality of the NPET is monitored throughout assembly of the 60S subunit, and errors in its construction can be propagated through rRNA.

\section{Results}

The uL4 tunnel domain is necessary for $60 \mathrm{~S}$ assembly. Previously, the tunnel domain (TD) of uL4 was shown to be necessary for $60 \mathrm{~S}$ subunit assembly ${ }^{12}$. However, its precise function during ribosome biogenesis remains unclear. Because the bilobal TD of uL4 (amino acids 63-87) forms constriction sites in the NPET and has more recently has been shown to interact with a number of tunnel-probing ribosome assembly factors ${ }^{15-17,21,22}$, we wanted to investigate its specific role in $60 \mathrm{~S}$ subunit assembly in greater detail. To this end, we have characterized multiple strains expressing different mutations or full deletions of the uL4 TD. We focused on the effects of the rpl4 $463-87$ mutation because it removes both constriction sites from the NPET (Fig. 2a, b). To assay effects of the rpl4 $\Delta 63-87$ mutation, we expressed the mutant uL4 protein from a plasmid in a strain conditional for expression of endogenous wild-type uL4 (GAL-RPL4). This strain grows at wild-type rates on galactosemedium but fails to grow on glucose-medium where only the mutant protein is expressed.

In order to determine which stages of the $60 \mathrm{~S}$ subunit assembly pathway are affected in the rpl4 $463-87$ mutant, we assayed prerRNA processing using primer extension and northern blotting. Compared to the wild-type strain, the rpl4 $463-87$ mutant accumulated both $27 \mathrm{SB}$ and $7 \mathrm{~S}$ pre-rRNAs, which normally undergo processing during late nucleolar and nucleoplasmic stages of 60S assembly (Supplementary Fig. 1a). To confirm that these middle steps of $60 \mathrm{~S}$ subunit assembly are affected in the rpl4 $\Delta 63-87$ mutant, we assayed localization of pre-60S subunits using uL23-eGFP (rpL25), a reporter of both mature and pre-60S subunits, and Nop1-mRFP, a nucleolar marker. Relative to the wild-type strain, we observed accumulation of pre-60S subunits in the nucleolus and nucleoplasm in the rpl4 $\Delta 63-87$ mutant (Supplementary Fig. 1b). These results demonstrate that the uL4 TD is necessary for late nucleolar and nucleoplasmic stages of $60 S$ subunit assembly prior to pre-60S export from the nucleoplasm. 
a

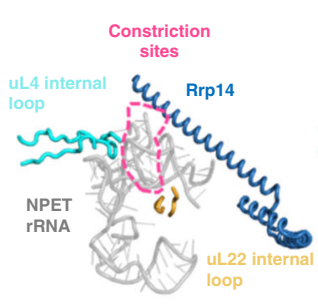

Nsa1 State 2

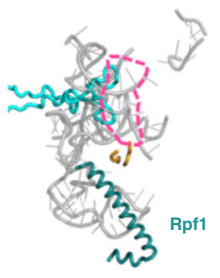

Nsa1 State C

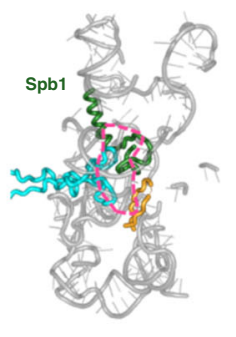

Nsa1 State E

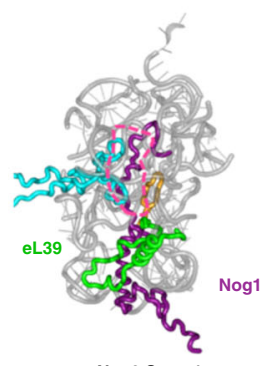

Nog2 State 1

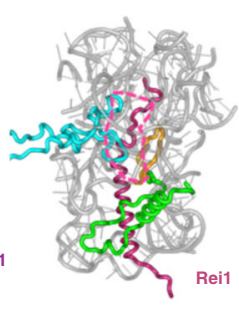

Arx1 Particle

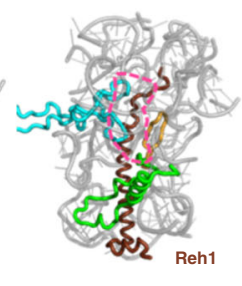

Nmd3 Particle

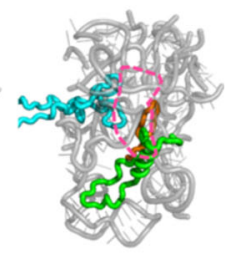

Mature tunnel b

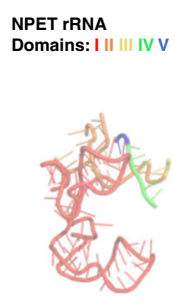

Nsa1 State 2

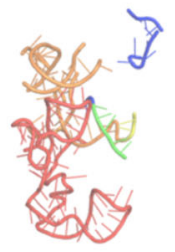

Nsa1 State C

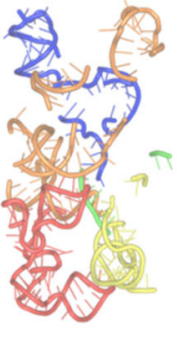

Nsa1 State E

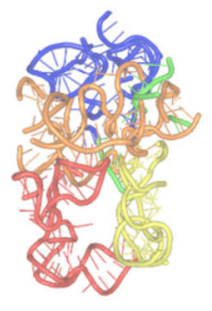

Nog2 State 1

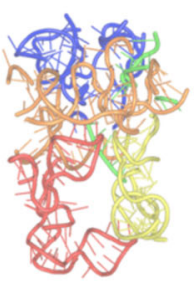

Arx1 Particle

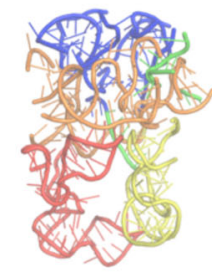

Nmd3 Particle

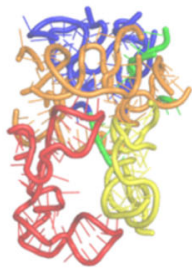

Mature tunnel

Fig. 1 Ordered stages of NPET assembly. a Cryo-EM structures of preribosomes revealed that the NPET undergoes distinct stages of assembly. The Nsa1 state 2 particle (PDB: 6COF) contains Rrp14 (sky blue) associated with an NPET precursor. The uL4 (cyan) and uL22 (bright orange) internal loops can be seen but the tunnel domain (TD) of uL4 is not yet visible. The Nsa1 state C particle (PDB: 6EM1) displays Rpf1 (teal green) in an incomplete NPET. The TD of uL4 (cyan) is now visible at this stage. The Nsa1 state E particle (PDB: 6ELZ) contains a more complete NPET. The CTD of Spb1 (dark green) can be seen toward the entrance of the NPET. In Nog2 particles (PDB: 3JCT), a nearly mature NPET, now containing eL39 (green), can be visualized with the CTD of Nog1 (dark purple) stretching almost the entire length of the NPET. The Arx1 (PDB: 5APN) and Nmd3 (PDB: 5H4P) particles reveal no further NPET rRNA maturation but show that the CTDs of Rei1 (dark pink) and Reh1 (brown), respectively, now occupy the NPET. b Five domains of rRNA secondary structure make up the NPET. In Nsa1 state C, the NPET contains mostly domains I (red) and II (orange). A small portion of domain IV (green) can also be seen and domain $\mathrm{V}$ (blue) has not yet folded into the NPET. In Nsa1 state E, domain III (yellow) has folded into a stable conformation to join the NPET. In Nog2 state 1, domain $V$ has stably folded into the NPET while a portion of domain II has also folded into a near mature state. Minor rearrangements in NPET rRNA can be observed in Arx1, Nmd3, and mature particles (PDB: 4V88). For clarity, only NPET-relevant portions of proteins and rRNA are shown.

The tunnel domain of uL4 stabilizes rRNA. Because our assays thus far suggest a defect during late nucleolar/nucleoplasmic stages of $60 \mathrm{~S}$ assembly, we used the late nucleolar/nucleoplasmic acting AF Nog2 as bait to affinity-purify particles from this stage of assembly. We performed cryo-EM on these Nog2-associated pre-60S particles, which enabled observation of both small and dramatic changes in rRNA and protein conformation that would otherwise be undetectable by other methods.

Previously, cryo-EM structures of wild-type Nog2-associated particles revealed three distinct consecutive assembly intermediates during middle stages of $60 \mathrm{~S}$ subunit assembly: $\operatorname{Nog} 2$ states 1,2 , and 3. Nog2 state 1 particles contain a prerotated $5 \mathrm{~S} R \mathrm{RP}, \operatorname{Nog} 2$ state 2 contains a rotated 5S RNP along with AFs Sdal, the Rixl complex, and Rea ${ }^{23}$, while Nog2 state 3 contains a rotated, near mature $5 \mathrm{~S}$ RNP and has released Sdal, the Rixl subcomplex, Real, and Rsa4. Therefore, using Nog2 as bait enabled us to assess the progress of 60 S subunit assembly during late nucleolar and nucleoplasmic stages.

Through cryo-EM 3D classification, we obtained seven states for the rpl4 $\Delta 63-87$ mutant particles, which we refer to as classes R1-R7 (Supplementary Fig. 2a, b) As expected, density for the deleted TD of uL4 could not be observed in any of these classes. Classes R3 and R4 comprise a small fraction of the particles $(10 \%)$, and represent intermediates inhibited during early steps of $60 \mathrm{~S}$ subunit maturation (Supplementary Fig. 3a, b) (see Discussion). The R1 and R2 particles (27\% and $12 \%$, respectively) closely resemble Nog2 state 1 particles, containing a prerotated $5 \mathrm{~S}$ $\mathrm{RNP}$, and thus have progressed beyond the stages represented by the R3 and R4 particles. The R7 class (10\%) is R1-like but lacks densities for nearly the entire domains III and IV of $25 \mathrm{~S}$ rRNA and appears to represent R1 particles undergoing turnover (Supplementary Fig. 3e). Class R6 particles contain a rotated 5S RNP and resemble Nog2 state 3 (Arxl particles) (Supplementary Fig. 3d), but also lack densities for several rRNA helices underneath the L1 stalk. Thus, R6 particles appear to be blocked later than class R1, after rotation of the 5S RNP. Finally, class R5 resembles R6 but, lacks densities for nearly the entire domains III, $\mathrm{IV}$, and $\mathrm{V}$ of $25 \mathrm{~S}$ rRNA and therefore may represent R6 particles undergoing turnover (Supplementary Fig. 3c).

We first focused on the preribosomes in classes R1 and R2 because they are the most abundant and stable mutant particles. The structures of these R1 and R2 particles were solved at a resolution of 3.2 and $3.3 \AA$ (Supplementary Fig. 2c, d). In classes R1 and R2, no density could be observed for the NPET-occupying RP eL39, the Nog1 CTD, or AF Arx1 that binds to the NPET exit platform (Fig. 2c, d). This indicates that lack of the L4 TD causes significant disruption in the composition of the immature NPET.

Several other protein components were also found to be missing or flexible in both classes R1 and R2 (Fig. 2d). We could not observe densities for Bud20, Nug1, and Cgr1. While Cgr1 is known to be necessary for $5 \mathrm{~S}$ RNP rotation ${ }^{24}$, it is not always detected in relevant pre-60S particles, even in wild-type Nog2 particles ${ }^{17}$. This suggests that Cgr1 may be loosely bound to pre605 subunits and may explain why it cannot be observed in rpl4 $\Delta 63-87$ mutant particles. 
a

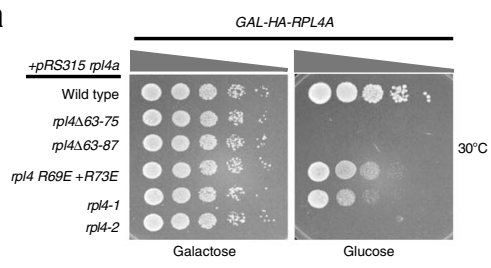

b

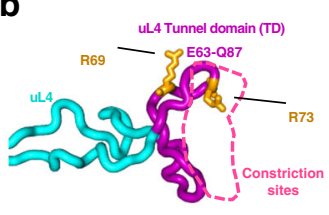

C

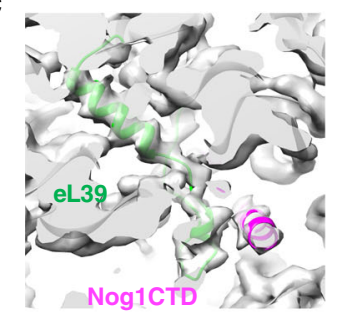

Nog2 state1

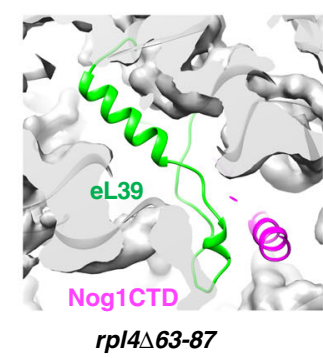

Class R1

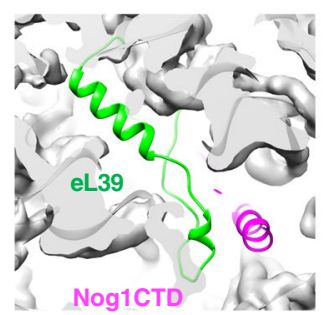

rpl4 $\Delta 63-87$

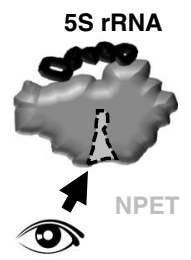

d

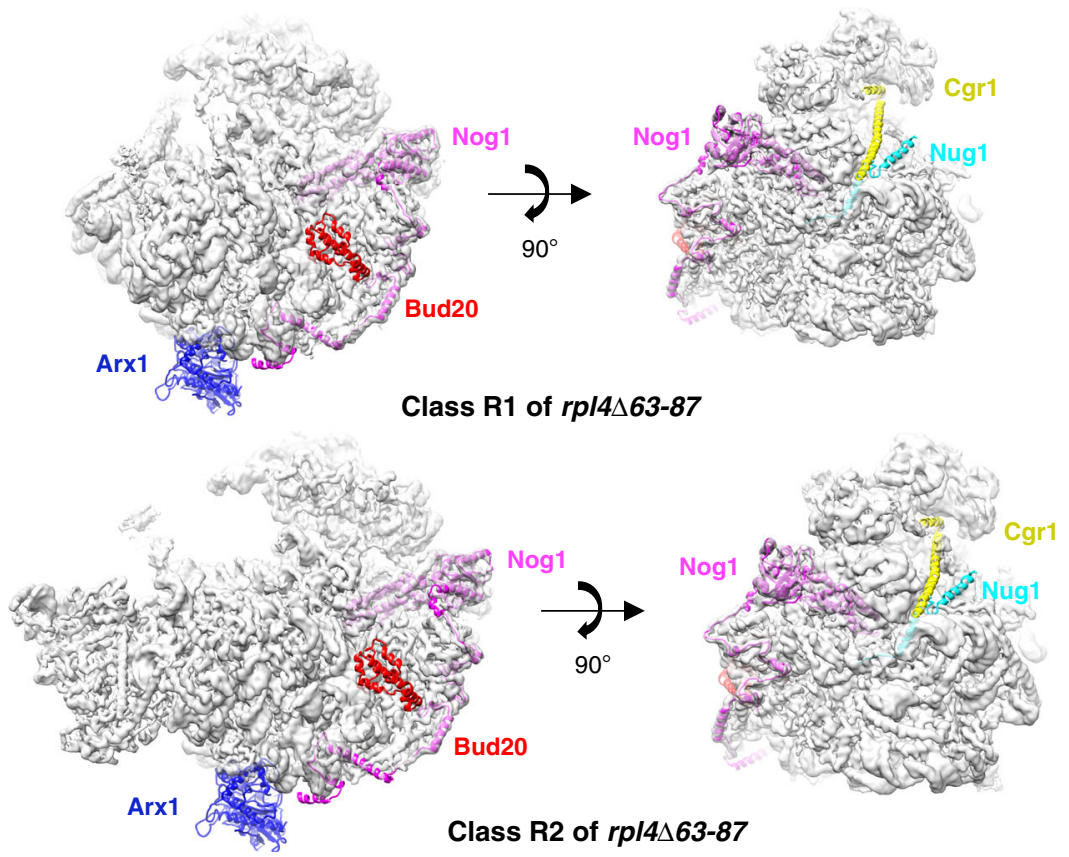

Fig. 2 Cryo-EM of $r$ pl4 $\triangle$ 63-87 Nog2-associated mutant particles. a Deletion of the uL4 tunnel domain (aa 63-87) is lethal. Each strain contains GAL-RPL4 that can be turned off by shifting cells from galactose to glucose-medium, and a plasmid that is constitutively expressing an rp/4 mutant allele. The rp/4-1 and rp/4-2 mutantions are alanine scans of residues 63-68 and 69-74, respectively. Serial dilutions (1:10 to 1:10,000) of cultures were pipetted onto selective medium containing either galactose or glucose, incubated at $30^{\circ} \mathrm{C}$, and imaged after 3 days. b Diagram of the internal loop of uL4 (cyan), including the TD (purple) that extends into the NPET to help create the constriction sites (dotted pink line). Indicated arginine residues (orange) were mutated to glutamate. The TD forms the constriction sites in the NPET (pink dotted line). c Close up view of the exit of the NPET in wild-type Nog2 state 1 and rp/4 663-87 classes R1 and R2. Gray densities are not present in classes R1 and R2 in the spaces where eL39 and the Nog1 CTD should be. Thus, the exposed cartoon model indicates missing components. d View of rpl4 $463-87$ classes R1 and R2 from the subunit interface (left) and solvent side (right). Densities are fitted to the atomic model of wild-type Nog2 state 1 (PDB: 3jct). Exposed cartoon models of Arx1 (blue), the Nog1 CTD (magenta), Bud20 (red), Cgr1 (yellow), and Nug1 (cyan) represent missing densities for each respective protein.

We next looked at effects of the rpl4 $\Delta 63-87$ mutation on the structure of pre-rRNA in Nog2 particles. Although class R2 exhibits wild-type rRNA conformations, significant shifts in rRNA helices 68-69 and 74-79 could be seen in class R1 (Fig. 3a, b). Further analysis of the atomic model of class R1 revealed how a misassembled NPET could cause these rRNA conformational changes (Fig. 3c). These aberrant shifts begin with a loss of the interaction between the TD of uL4, the Nog1 CTD, and the linker between rRNA helices 73 and 74 (Fig. 3d).
Recent cryo-EM structures further suggest the importance of this interaction ${ }^{25}$. H74 is displaced $\sim 4 \AA$ relative to the wild-type conformation, indicating that this portion the NPET is improperly assembled (Fig. 3e). The conformational change of H74 affects the adjacent H75, which can be seen shifted up to $\sim 33 \AA$ from its native position in Nog2 state 1 (Fig. $3 \mathrm{e}$ ). In this aberrant position, $\mathrm{H} 75$ clashes with $\mathrm{H} 68$ and displaces it up to $\sim 62 \AA$ toward the $5 \mathrm{~S}$ rRNA (Fig. $3 \mathrm{f}$ ). H68 is the binding site for AF Sda1, which is important for rotation of the 5S RNP in Nog2 
a

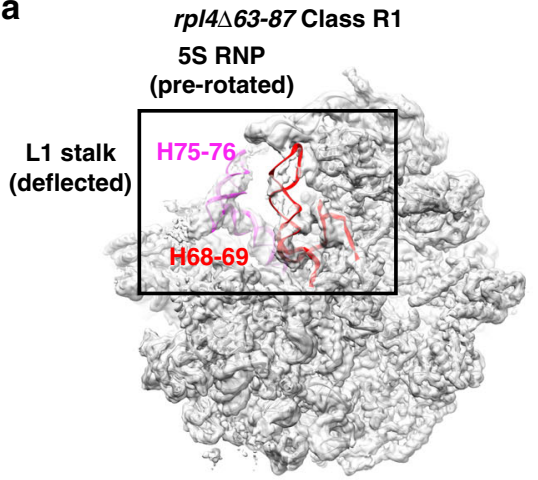

b

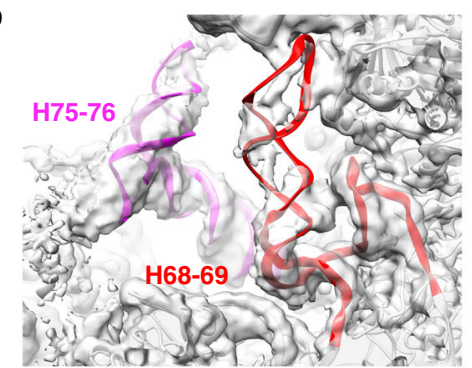

C
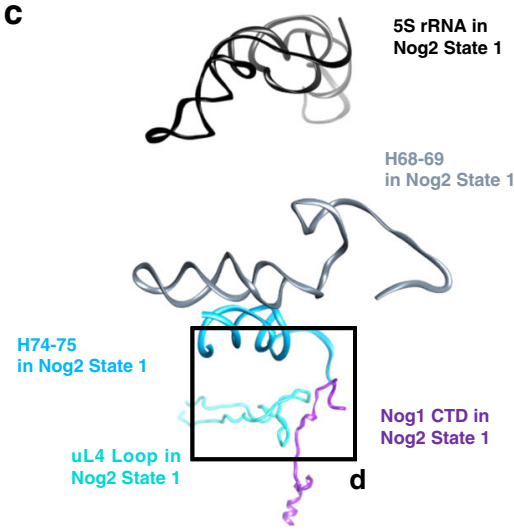

Nog2 State 1
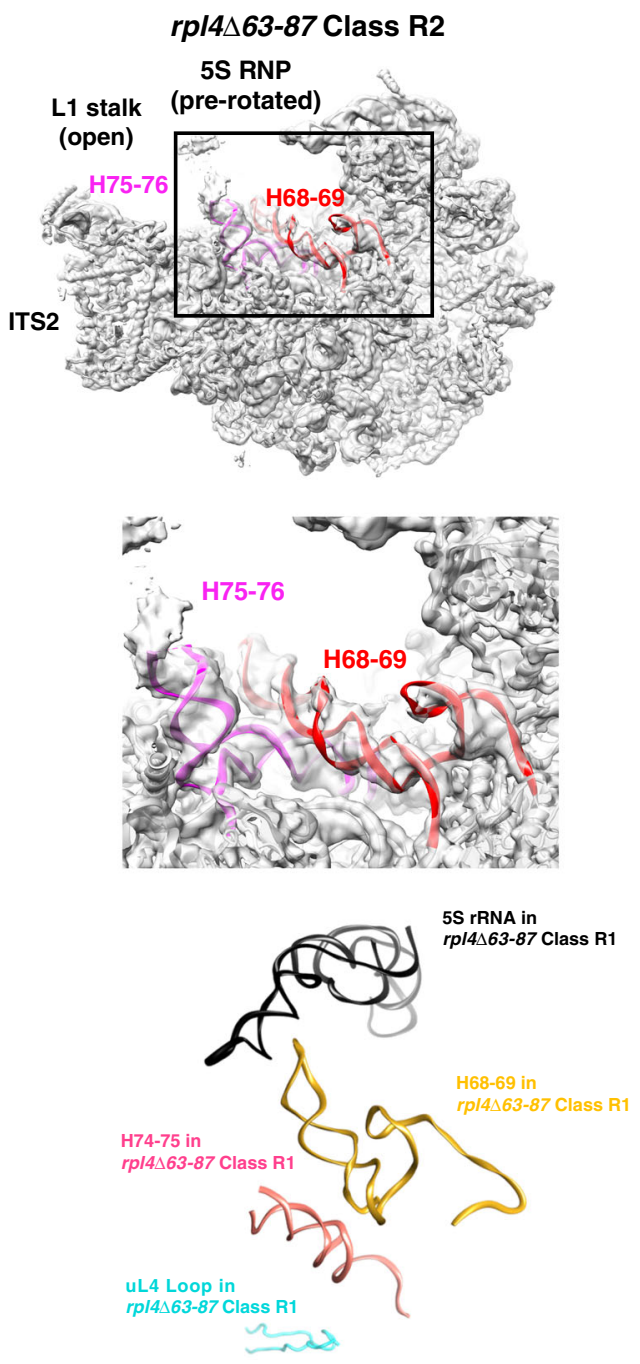

d

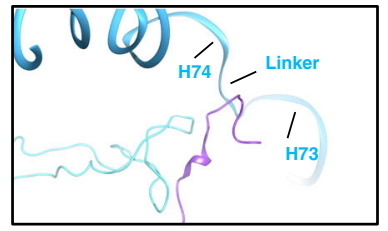

e

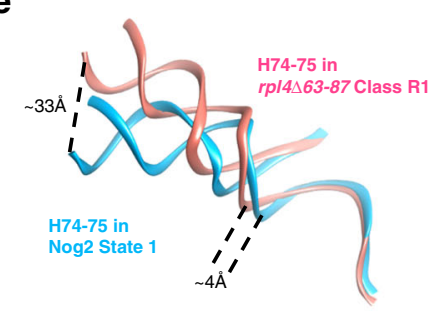

rpl4 $\Delta 63-87$ Class R1

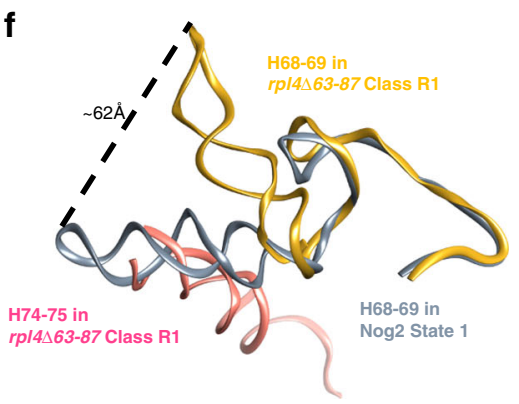

Fig. 3 Aberrant conformations of rRNA helices in rpl4 $\Delta$ 63-87 mutant particles. a Two major density maps (R1 and R2 particles, shown in light gray) obtained from Nog2-associated $r$ /4 $\Delta 63-87$ particles are overlaid with their respective atomic models. $\mathbf{b}$ Enlarged view of the conformational changes in of rRNA helices from the two major classes of the rp/4 $463-87$ mutant. Compared with wild-type Nog2-particles (state 1), H75-76 (magenta) and H68-69 (red) have undergone dramatic conformational changes in class R1 but not in class R2. c rRNA helices from the atomic model of wild-type Nog2 state 1 particles are compared with those in the atomic model of the rp/4 $\Delta 63-87$ mutant class R1. Positions of relevant rRNA helices in relation to uL4 and Nog1 in the NPET in RPL4 wild-type (left) and rp/4 $463-87$ mutant (right) Nog2 particles. Helix 74-75 (light blue for wild-type and hot pink for mutant) extend into the NPET. H74 is located in close proximity to the uL4 TD (cyan) and the CTD of Nog1 (dark purple). H74-75 are located below H68-69 (gray for wild-type and gold for mutant). The $5 \mathrm{~S}$ rRNA (black) is shown to provide a frame of reference. $\mathbf{d}$ Inset showing the interaction of the TD of uL4, the Nog1 CTD, and the rRNA linker between $\mathrm{H} 73$ and H74. e H74-75 from rp/4 $463-87$ mutant particles (hot pink) are shifted relative to those in wild-type Nog2 state 1 particles (light blue). $\mathrm{H} 74$ is displaced up to $\sim 4 \AA$ while $\mathrm{H} 75$ is shifted up to $\sim 33 \AA$. $\mathbf{f H 7 5}$ in rpl $4 \Delta 63-87$ mutant particles is shifted to an aberrant position (hot pink) that clashes with the native position of $\mathrm{H} 68$ (gray). This displaces $\mathrm{H} 68$ in rpl4 $\Delta 63-87$ mutant particles (gold), causing it to shift up to $\sim 62 \AA$. 
particles and acts as an intersubunit bridge in mature wild-type ribosomes. Once structured in Nog2 state 1, this helix remains stably docked throughout middle and later stages of $60 \mathrm{~S}$ subunit assembly $8,17,21,22,26$. Therefore, deviations from this stable position of $\mathrm{H} 68$, as seen in class $\mathrm{R} 1$, likely have negative consequences as a result of improper NPET construction during the assembly of 605 subunits.

Consistent with the displaced H74-75, the 3-way junction of rRNA helices 75, 76, and 79 is affected in class R1. Specifically, the L1 stalk (H76 and uL1) adopts an unusual, deflected conformation not observed in any previously characterized wild-type particles (Supplementary Fig. 4) and H79 is unstructured. As a result, the internal loop of uL15, which interacts with $\mathrm{H} 75$ in Nog2 state 1, cannot be visualized. Likewise, the Nterminus of eL8, which interacts with the 3-way junction of $\mathrm{H} 75$, $\mathrm{H76}$, and H79 and was previously shown to be necessary for middle stages of $60 \mathrm{~S}$ subunit assembly ${ }^{27}$, is also flexible (Supplementary Fig. 5a-d). These observations further characterize the perturbance of this 3-way junction as a result of improper NPET construction.

A misassembled NPET can block Sdal binding to the pre-60S. To help clarify the consequences of the aberrant rRNA conformations observed in rpl4 $\Delta 63-87$ mutant pre-60S subunits, we utilized SDS-PAGE, western blotting, and iTRAQ mass spectrometry to analyze the protein composition of these particles.

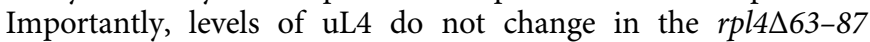
mutant preribosomes compared to wild-type, indicating that the mutant uL4 protein is stable and able to efficiently assemble into pre-60S subunits. However, we did observe a number of changes in amounts of other proteins in the mutant preribosomes. Most striking was that levels of Sda1 decreased and levels of Rpf2 and Rrs1 increased (Fig. 4a, b). In wild-type cells, the AFs Rpf2 and Rrs1, which are bound to the 5S RNP, are thought to exit from Nog2 particles before Sda1 binds to pre-60S subunits ${ }^{28}$. Thus, Sda1 fails to associate with a significant fraction of pre-60S subunits in this mutant, and Rpf2 and Rrs1 fail to exit from preribosomes. Furthermore, amounts of downstream AFs Rea1, and the Rix1 complex (Rix1, Ipi1, and Ipi3), which depend on Sda1 for recruitment onto pre-60S subunits and are required to stabilize 5S RNP in its rotated state, also are decreased relative to wild-type (Fig. 4b). Consistent with a failure of Rea1 to enter assembling pre-60S subunits, levels of Rsa4, which is removed by Rea1, are increased.

The C-terminal domain of Nog1 facilitates $60 \mathrm{~S}$ assembly. Since the Nog1 CTD is in close contact with the TD of uL4 in wild-type cells and fails to enter the NPET in rpl4 $\Delta 63-87$ mutant particles, we wanted to investigate whether deleting the Nog1 CTD results in similar or different $60 \mathrm{~S}$ subunit assembly defects compared to those observed in the rpl4 $\Delta 63-87$ mutant. Thus, we generated a series of genomic mutations to sequentially truncate the CTD of Nog1 (Fig. 5a and Supplementary Fig. 6a). We also constructed the triple mutant nog1 $\Delta 595-647$ rei1 $\Delta 341-393$ reh1 $1880-432$ (nog1 $\Delta C$ reil $\Delta C$ reh1 $\Delta C$ ) lacking the NPET-occupying sequences of Nog1, Rei1, and Reh1. As an orthogonal approach to deleting Nog1 sequences inserted into the tunnel, we constructed a strain expressing Nog1 fused to GFP at its C-terminus (NOG1-GFP). Because an immature NPET is already formed before the Nog1 CTD enters the tunnel (Fig. 1a), this bulky GFP tag must prevent insertion of the Nog1 CTD into the tunnel.

All of these mutants and the NOG1-GFP strain exhibit an extreme cold-sensitive growth defect (Fig. 5a). In contrast, an analogous C-terminal truncation of Reil has been shown to have minimal effects on cell growth at all temperatures, while the complete absence of Reh1 has no obvious growth defect ${ }^{22,29}$. To examine whether this cold-sensitivity reflects a defect in $60 \mathrm{~S}$ subunit assembly, we used sucrose gradient fractionation to assay amounts of free ribosomal subunits, $80 \mathrm{~S}$ monosomes, and polyribosomes in extracts prepared from each strain. In each case, levels of free $60 \mathrm{~S}$ ribosomal subunits are decreased relative to $40 \mathrm{~S}$ subunits, polyribosomes are decreased, and halfmer polyribosomes are present, indicating a defect in production of $60 \mathrm{~S}$ subunits (Supplementary Fig. 6b).

To determine which interval of 60S subunit assembly is perturbed in each mutant, we examined pre-rRNA processing. When cells were shifted to $16^{\circ} \mathrm{C}$ for $5 \mathrm{~h}, 27 \mathrm{SB}$ pre-rRNA accumulates in all mutant strains relative to the wild-type NOG1 yeast strain (Supplementary Fig. 6c). Together, these results indicate that the presence of the CTD of Nog1 in the NPET is important during late nucleolar and nucleoplasmic stages of $60 \mathrm{~S}$ subunit assembly.

The C-terminal domain of Nog1 scaffolds uL4. We performed cryo-EM of Nog2-containing particles affinity purified from the nog $1 \Delta C$ reil $\Delta C \operatorname{reh} 1 \Delta C$ mutant, shifted to $16^{\circ} \mathrm{C}$ for $5 \mathrm{~h}$. We focused on this triple mutant because it exhibited the most severe growth defect and we expected that the CTD's of Reil and Reh1 might partially rescue defects of a nog1 $\Delta 595-647$ mutant. We obtained structures of four stable intermediates at resolutions ranging from 3.0 to $4.3 \AA$, which we refer to as classes N1-N4 (Supplementary Figs. 7 and 8). The most striking observation from these structures is that the interior of the NPET in all four nog $1 \Delta C$ reil $\Delta C$ reh $1 \Delta C$ particle classes lacks density for the TD of uL4 (Fig. 5b). This result indicates that the presence of the Nog1 CTD in the NPET is required to stabilize the TD of uL4. In contrast to the rpl4 $\Delta 63-87$ mutant particles, eL39 was present in classes N1-N4 (Fig. 5b). Classes N1 and N2 closely resemble class $\mathrm{R} 2$ from the $r p l 4 \Delta 63-87$ mutant, except that the ITS2 structure is absent in class N2 (Fig. 5c). This latter observation is consistent with previous findings that removal of ITS2 can occur independently from other remodeling events such as 5S RNP rotation $^{30,31}$. Class $\mathrm{N} 3$ particles resemble class $\mathrm{R} 1$ from the rpl4 $\Delta 63-87$ mutant, including the aberrant rRNA rearrangements, deflected L1 stalk, and absence of Sda1 (Fig. 6a, b). However, while class R1 makes up about $27 \%$ of total particles obtained from the rpl4 $\Delta 63-87$ mutant, class N3 only comprises about $7 \%$ of total particles recovered from the nog $1 \Delta C$ reil $\Delta C$ reh $1 \Delta C$ mutant. Most notable is class N4. Unlike any classes observed in the rpl4 $\Delta 63-87$ mutant, class N4 represents improperly assembled Nog2 state 2 particles; it displays weak density for the Rixl complex and lacks density for Rea1. Consistent with inefficient recruitment of the Rix1 complex and Rea1, the Rea1dependent removal of Rsa4 has not yet occurred in class N4 (Fig. 7c). These results indicate that the nog $1 \Delta C$ reil $\Delta C$ reh $1 \Delta C$ mutant is distinct from the rpl4 $\Delta 63-87$ mutant. In this mutant, a smaller fraction of intermediates appears to be blocked at the stage of Nog2 state 1, and the second block appears to be at the state 2 stage, rather than the state 3 stage, of Nog2 particles. These differences between the rpl4 $\Delta 63-87$ mutant and the nog $1 \Delta C$ rei1 $\Delta C$ reh $1 \Delta C$ mutant may in part result from the absence of the TD of uL4 in the former, versus its being present but in a flexible conformation in the latter.

To further characterize the differences between $r p l 4 \Delta 63-87$ and nog $1 \Delta C$ reil $\Delta C$ reh $1 \Delta C$ mutant particles, we assessed the protein composition of Nog2-associated pre-60S subunits using SDSPAGE, western blotting, and iTRAQ mass spectrometry. Purifications were done using wild-type or mutant cells grown at $30^{\circ} \mathrm{C}$ and then shifted to $16^{\circ} \mathrm{C}$ for five hours. SDS-PAGE followed by silver staining or western blotting indicated that 
a

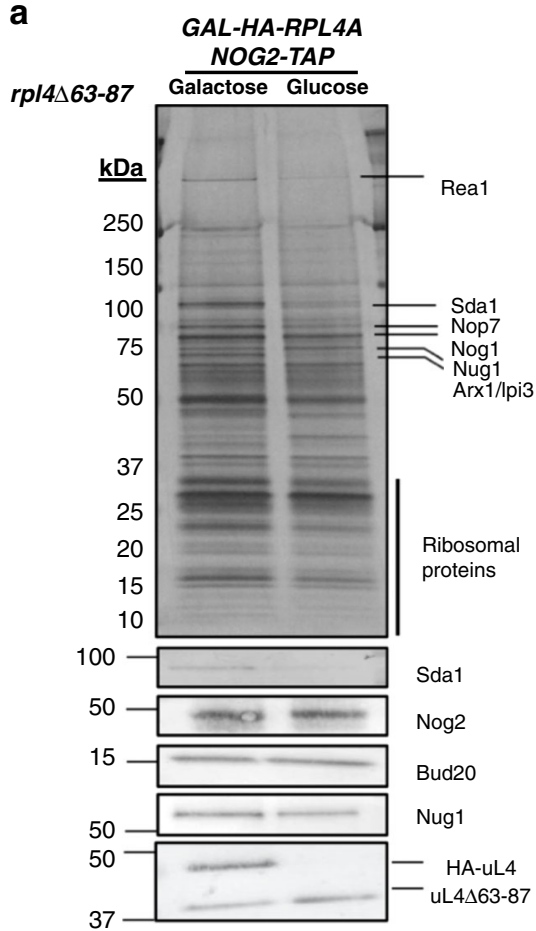

b

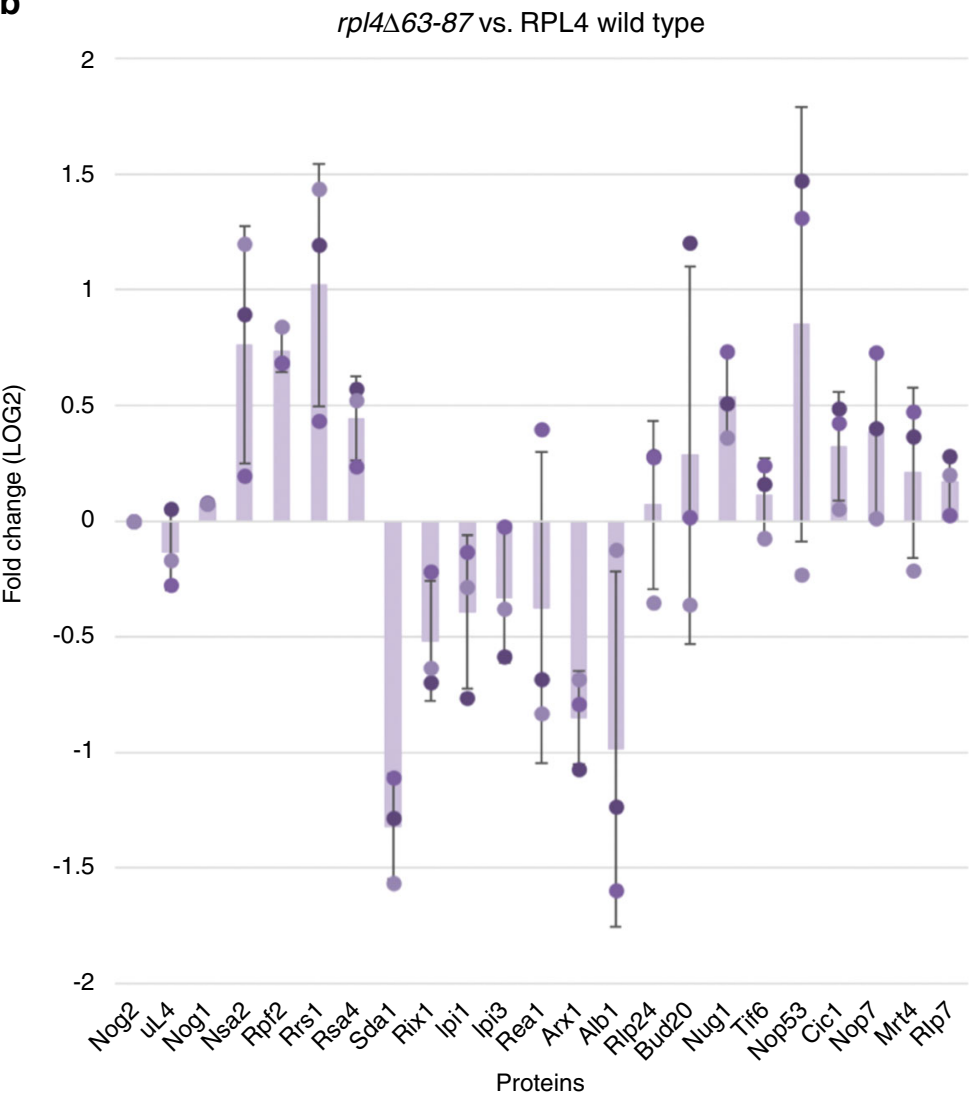

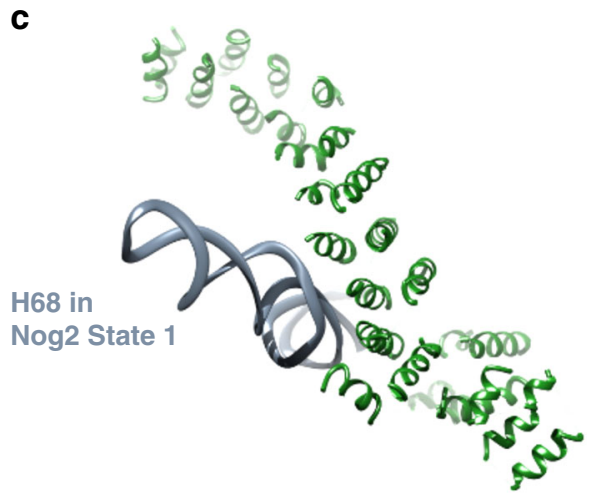

Nog2 State 1

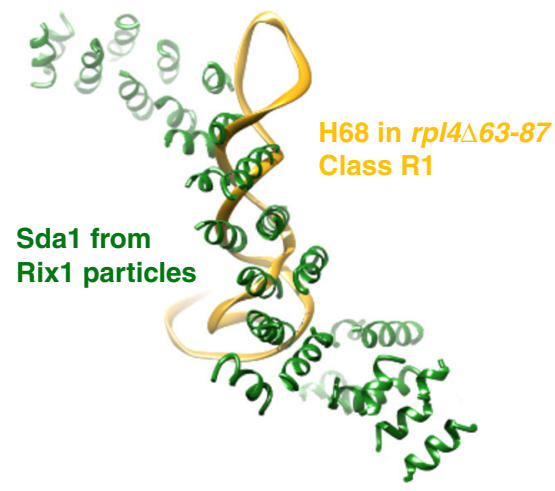

rpl4 $463-87$ Class $\mathrm{R} 1$

Fig. 4 Deviations in rRNA structure in rpl4 $\Delta$ 63-87 mutant particles result in $\mathbf{H 6 8}$ clashing with the binding site of Sda1. a SDS-PAGE gels followed by silver staining or western blotting show proteins in Nog2-associated particles purified from mutant $r p / 4 \Delta 63-87$ cells. Labeled bands were identified using mass spectrometry. b Samples prepared as in panel a were scaled up and subjected to semi-quantitative iTRAQ mass spectrometry to assay changes in relative amounts of pre-60S subunit proteins. Proteins were labeled with iTRAQ reagents and compared to wild-type counterparts. Each bar represents a biological replicate. All protein levels are normalized to the bait protein, Nog2. Ratios are represented on a $\log _{2}$ scale. Error bars represent standard deviation $(n=3)$. c The AF Sda1 (dark green) from Rix1 particles superimposed over wild-type Nog2 state 1 particles (left) and rp/4 $\Delta 63-87$ mutant particles (right). H68 (gray for wild-type and gold for mutant) in rpl4 $663-87$ mutant particles clashes with almost the full length of observable Sda1.

deletion of the Nog1 CTD does not affect the stability or recruitment of Nog1 into pre-60S subunits (Fig. 7a). In contrast to the rpl4 $\Delta 63-87$ mutant, levels of Sda1, Rpf2, and Rrs1 do not change in nog $1 \Delta C$ reil $\Delta C$ reh $1 \Delta C$ mutant particles relative to wild-type cells (Fig. 7b). Despite the observation that both the exit of Rpf2 and Rrs1 and the entry of Sdal are unaffected in the nog $1 \Delta C$ rei1 $\Delta C$ reh $1 \Delta C$ mutant, levels of the Rixl complex and Rea1, which are required for stabilization of the 5S RNP in its rotated state, are consistently decreased relative to wild-type (Fig. 7b). These results indicate that, for reasons that are still not entirely clear, nog $1 \Delta C$ rei1 $\Delta C$ reh $1 \Delta C$ mutant pre-60S subunits are able to recruit Sdal but cannot recruit the other machinery necessary for $5 \mathrm{~S}$ RNP rotation as efficiently as wild-type particles. This unusual protein composition most closely reflects what was observed by cryo-EM of class $\mathrm{N} 4$ of the $\operatorname{nog} 1 \Delta \mathrm{C} \operatorname{re} 1 \Delta \mathrm{C} \operatorname{reh} 1 \Delta \mathrm{C}$ mutant particles (Fig. 7c). 
a

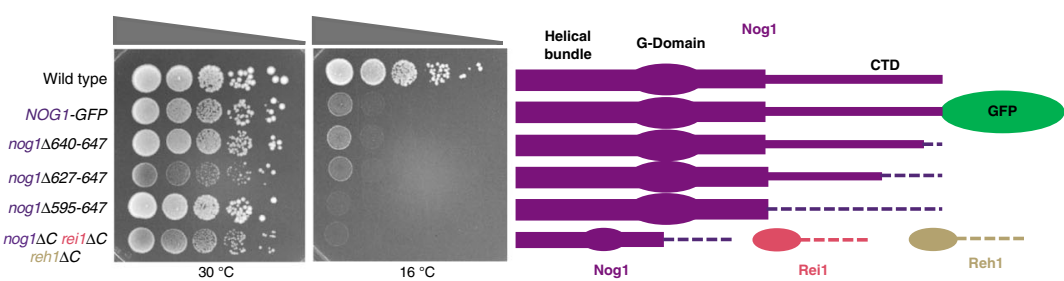

b

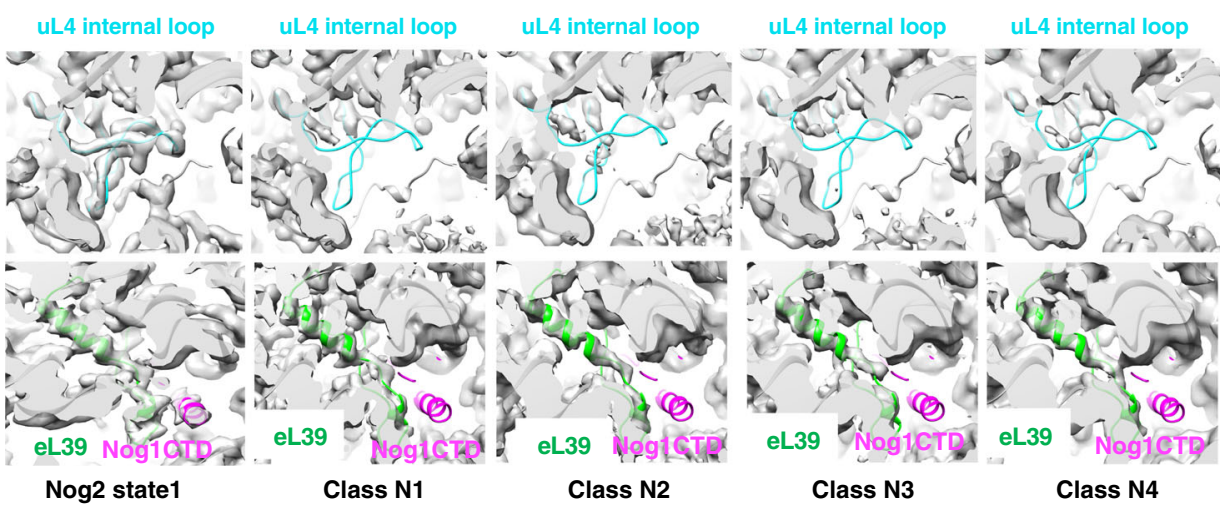

C
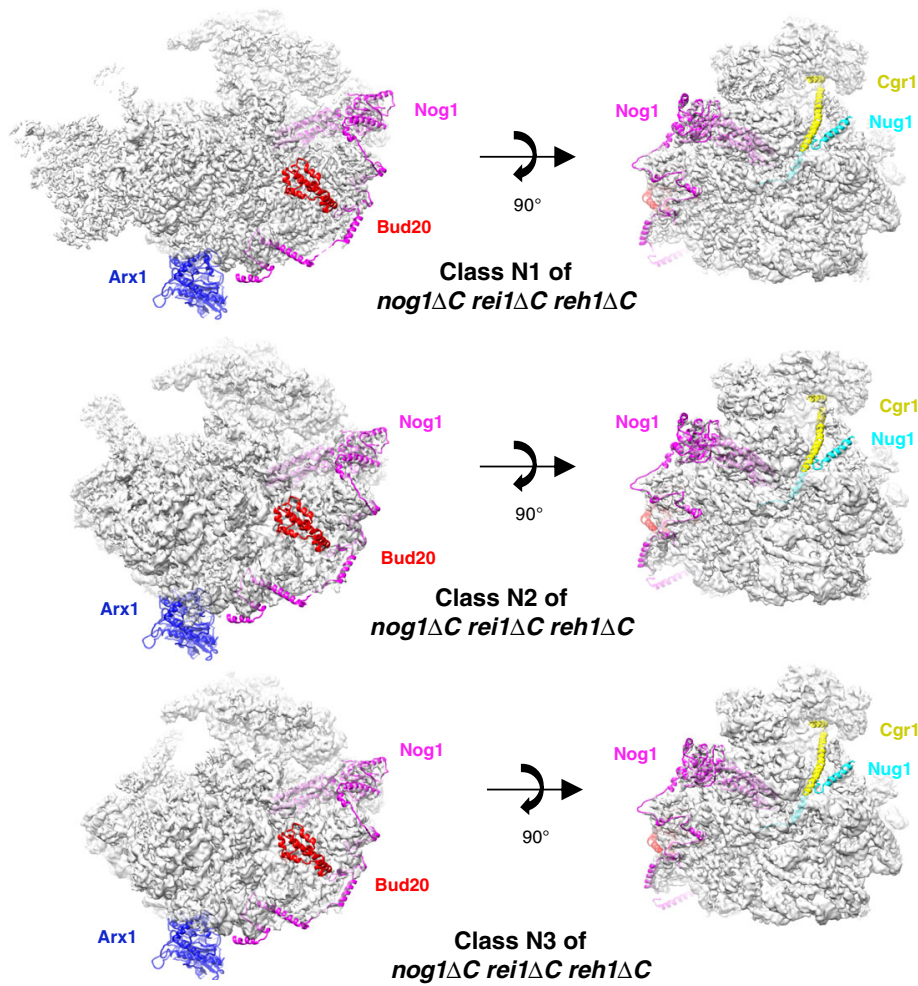

Fig. 5 The Nog1 CTD stabilizes the tunnel domain of uL4. a Serial dilutions (1:10 to 1:10,000) of NOG1 wild-type and nog1 mutant cultures were spotted onto solid medium containing glucose, and incubated at $30^{\circ} \mathrm{C}$ or $16^{\circ} \mathrm{C}$, for 8 days. Each construct is depicted in cartoon form (right). $\mathbf{b}$ Density maps of the wild-type Nog2 state 1 and N1-N4 mutant particles are aligned with the atomic model of wild-type Nog2 state 1 particles, displaying the interior of the NPET. Densities (gray) for the uL4 TD (cyan) are missing in the NPET in all four mutant classes (top). Densities for eL39 (green) can be visualized in classes N1-N4 but no densities for the Nog1 CTD (magenta) are visible (bottom). c View of nog1 $\Delta C$ rei1 $\Delta C$ reh1 $\Delta C$ classes N1-N3 from the subunit interface. Densities are fitted to the atomic model of wild-type Nog2 state 1 (PDB: 3jct). Exposed cartoon models of Arx1 (blue), the Nog1 CTD (magenta), Bud20 (red), Cgr1 (yellow), and Nug1 (cyan) represent missing densities for each respective protein.

\section{Discussion}

Here, we combined molecular genetics and biochemical approaches with high-resolution cryo-EM to uncover how construction of the NPET fits into the hierarchy of 60S ribosomal subunit assembly in yeast. Our work reveals that a misassembled NPET can delay or block at least three different stages of pre-60S subunit assembly and demonstrates interdependence between the TD of uL4 and the CTD of Nog1.

Previous work purifying Nog2-associated pre-60S particles from wild-type cells yielded three major stable assembly 
a

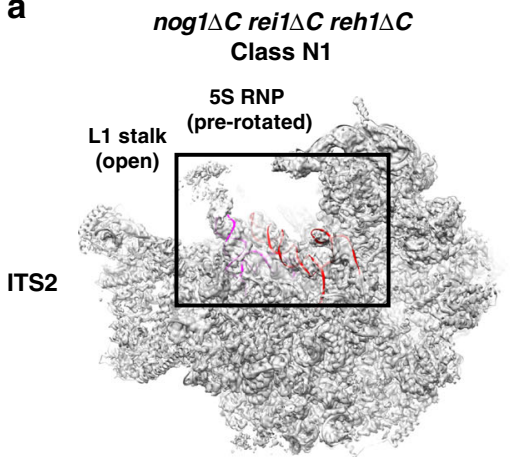

b

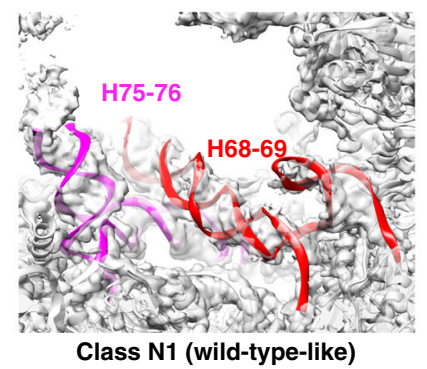

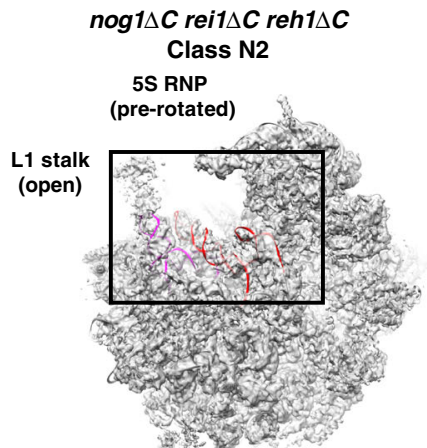
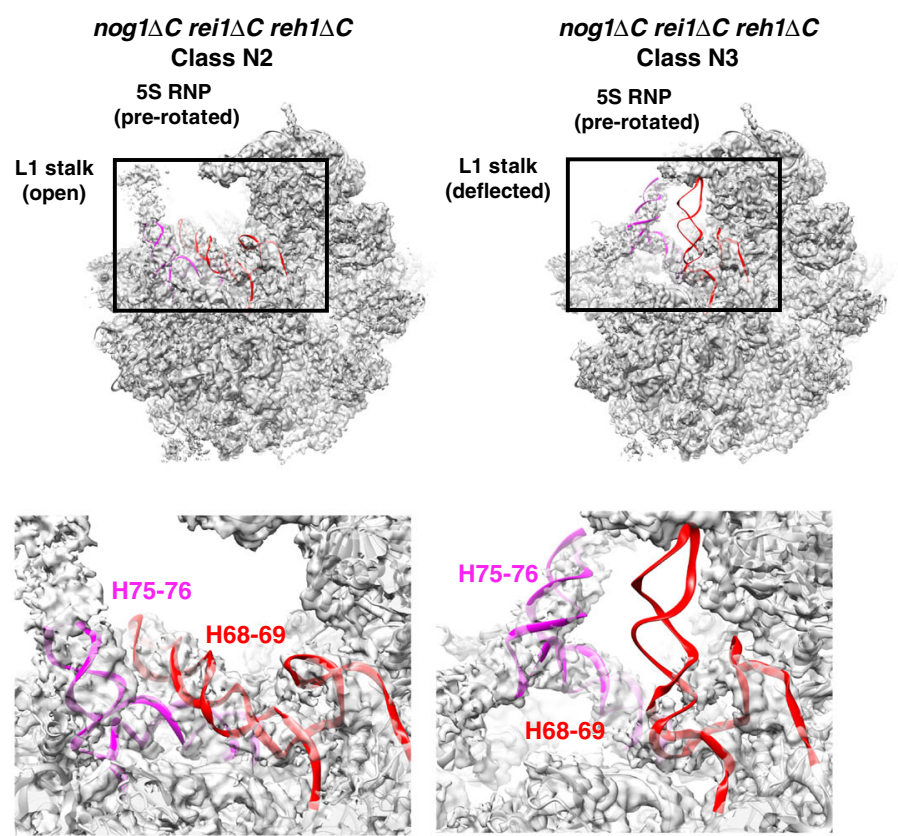

Class N2 (wild-type-like)

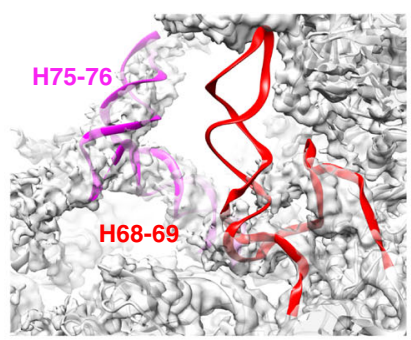

Class N3 (class R1-like)

Fig. 6 A minority of nog1 $\Delta C$ rei1 $\Delta C$ reh1 $\Delta C$ mutant pre-60S particles display aberrant rRNA conformations. a Densities of classes N1-N3 of nog1 $1 C$ rei1 $\Delta C$ reh1 $\Delta C$ mutant particles solved at a resolution of 3.0-6.0 $\AA$. Classes N1 and N2 are aligned with the atomic model of class R2, while class N3 is aligned with the atomic model of class R1. Note that ITS2 is missing from classes N2 and N3. b View of H68-69 (red) and H75-76 (magenta) from classes N1-N3.

intermediates (Nog2 states $1-3)^{17}$, with 5S RNP rotation occurring in Nog2 state 2. Our cryo-EM analysis of Nog2-associated pre-60S particles from the rpl4463-87 mutant revealed seven different particle states. Based on our data as well as others' data, we propose a model to explain the chronological progression and fate of each individual particle class through the 60S subunit assembly pathway (Fig. 8).

Using the atomic model of class R1 from the rpl4 $\Delta 63-87$ mutant, we were able to visualize several rRNA conformational changes that are apparently initiated by the instability of the linker between $\mathrm{H} 73$ and $\mathrm{H} 74$, which results in increased flexibility of H74 (Fig. 3c, 3d). In wild-type particles, H74 is one of the last rRNA helices in the NPET to undergo maturation (Fig. 1b). The absence of the UL4 TD results in a flexible H74, which appears to be sufficient to cause H75 to adopt an aberrant conformation. Consequently, H68 cannot remain stably docked on pre-60S subunits, and the 3-way junction of H75, H76, and H79 becomes destabilized, causing the L1 stalk to adopt a deflected position. Notably, densities for a shifted H75 could also be observed in classes R4 and R6 (Supplementary Fig. 9a). Consistent with this observation, densities for $\mathrm{H} 68$ are not visible in either class (Supplementary Fig. 9b), and the L1 stalk is deflected in class R4, the same as R1, indicating that L1 stalk maturation may be affected in early stages of $60 \mathrm{~S}$ assembly. In summary, we suggest that a flexible H74, which occurs as a consequence of an improperly formed NPET, can shift rRNA helices 75, 76, and 68 toward aberrant conformations that can affect several stages of 60 S subunit assembly.

Classes R3 and R4 resemble the early nucleolar intermediates Nsal state B and E, respectively, which and do not usually copurify with Nog2 particles ${ }^{16,17}$. However, class R4 contains density for the bait protein Nog2 and is therefore unlikely to be a contaminant. Nog2 may be entering pre-60S particles earlier due to their stalling 32 . This defect in early particles resulting from the absence of the TD of uL4 is consistent with the observation that the TD of UL4 is first visible (and therefore present in a stable form) in the NPET at this stage of wild-type 60S subunit maturation (Fig. 1a). Class R4 lacks densities for several AFs typically found in State E particles, including Noc3, Spb1, Brx1, and Ebp2. These AFs have most likely already associated and dissociated from pre-60S particles, while the AFs still remaining on particles (Erb1, Ytm1, and Has1) have not yet been released. Otherwise, failure of so many essential AFs to initially bind early assembly intermediates would result in their rapid turnover ${ }^{33}$. It remains unclear what the biological consequences of a deflected L1 stalk in class R4 may be. Therefore, classes R3 and R4 may represent on-pathway particles that are delayed in their progression to middle stages of assembly.

The rpl4 $463-87$ mutant particles that reach class R2 may subsequently become trapped in class R1, be unable to bind Sda1, and become destined for turnover (class R7). Alternatively, they might progress beyond the $5 \mathrm{~S}$ RNP rotation checkpoint to class R6. Because densities for H68 are not visible in class R6, we suspect that this flexible intersubunit bridge results in particles destined for turnover (class R5). However, in the case of R6, this flexibility of H68 may not arise until after 5S RNP rotation occurs (Fig. 8). Together, our data suggest that proper maturation of the NPET affects the stability of specific rRNA helices and keeps pre$60 \mathrm{~S}$ particles on-pathway. These mutant classes blocked at various stages may reflect the phenomenon of rerouting ribosome assembly to alternate pathways in response to stress $^{34,35}$.

Deletion of the TD of uL4 consistently resulted in more extreme phenotypes than those observed in nog1 CTD mutants. For example, the $r p l 4 \Delta 63-87$ mutant is lethal at all temperatures, whereas the nog1 CTD mutants exhibited severely reduced growth only at $16^{\circ} \mathrm{C}$ (Figs. 2a, 5a). The different extent to which maturation of pre-60S subunits is blocked in rpl4 compared to nog1 mutants may reflect the difference between the absence of 
a

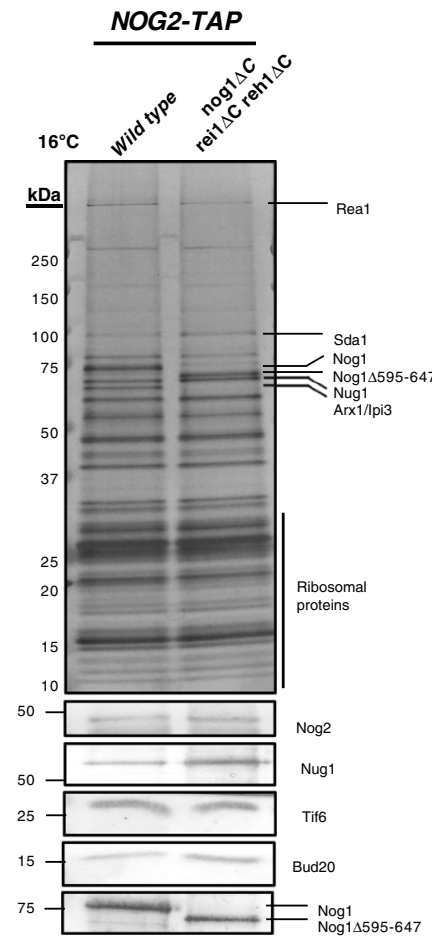

b

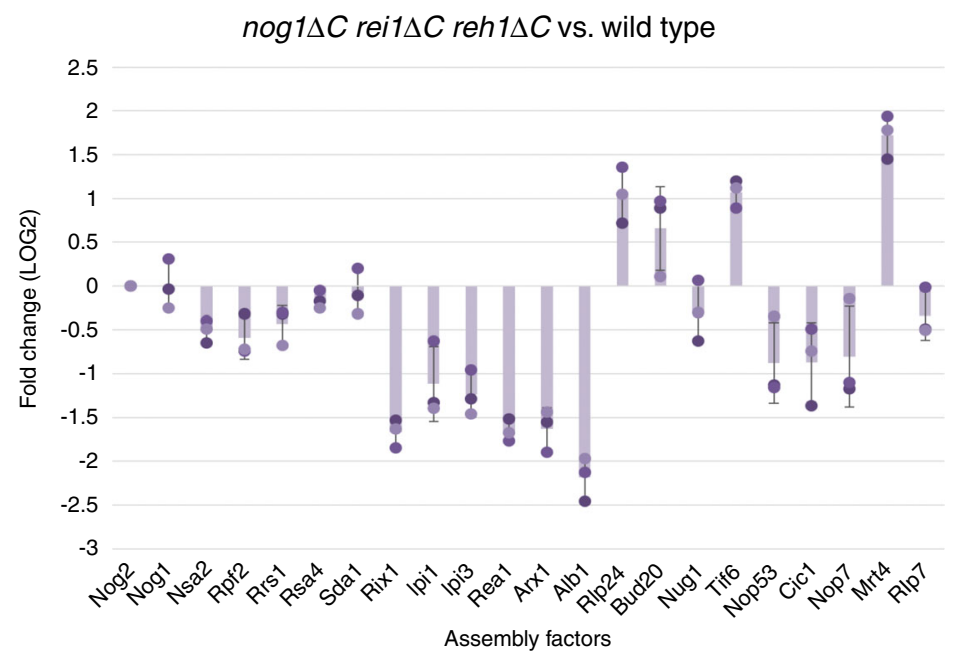

C
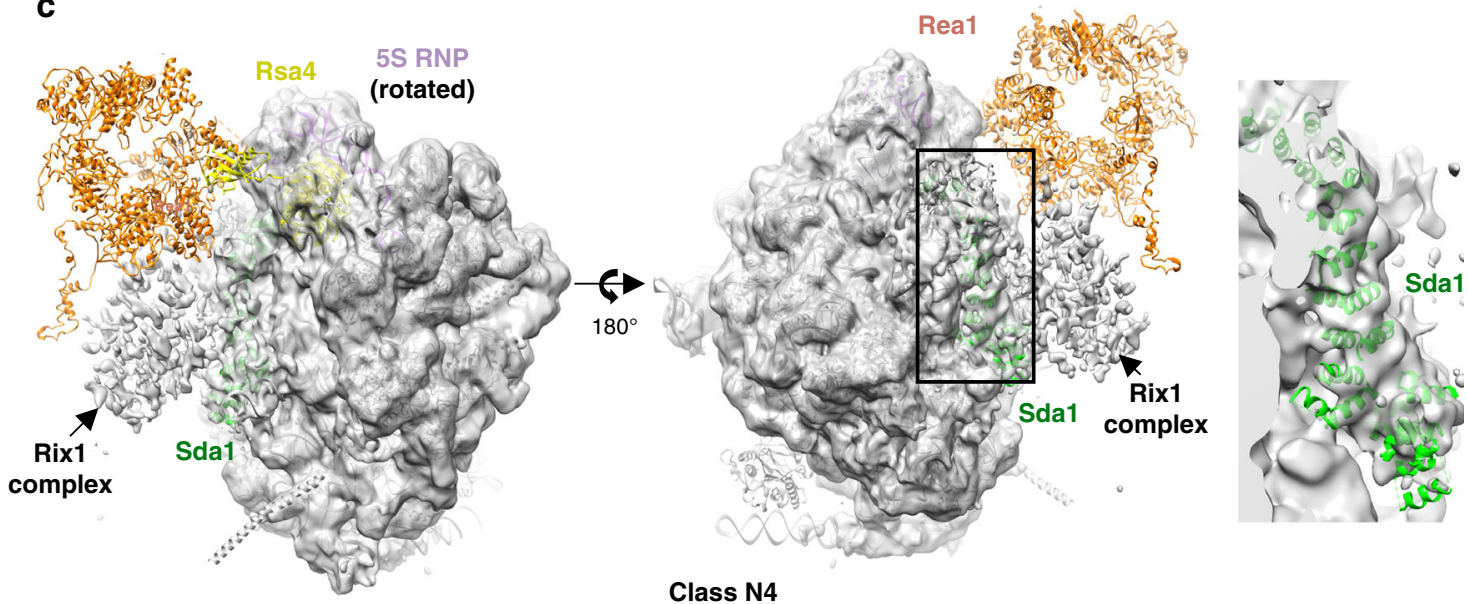

Class N4

Fig. 7 Truncation of the Nog1 CTD may affect closing of the $\mathbf{L 1}$ stalk. a SDS-PAGE of proteins in Nog2-associated preribosomes affinity purified from wild-type and nog $1 \Delta \mathrm{C}$ rei1 $\Delta \mathrm{C}$ reh1 $\Delta \mathrm{C}$ mutants shifted to $16^{\circ} \mathrm{C}$ for $5 \mathrm{~h}$. Proteins in labeled bands were identified by mass spectrometry. $\mathbf{b}$ Semi-quantitative ITRAQ mass spectrometry reveals differences in relative amounts of proteins labeled with iTRAQ reagents in Nog2-particles affinity purified from the nog $1 \Delta C$ rei1 $\triangle C$ reh1 $\Delta C$ mutant compared to those in wild-type particles. Each bar represents a biological replicate. All protein levels are normalized to the bait protein, Nog2. Ratios are represented on a $\log _{2}$ scale. Error bars represent standard deviation $(n=3)$. c Densities for class N4 are fitted to the atomic model of the Rix1 particle (PDB: 5fl8). Density for Sda1 (green) can be seen in the particles. The 5S RNP (purple) is in the rotated state in class N4. Weak density can be seen for the Rix1 complex, and Rea1 (orange) is almost completely missing. Rsa4 (yellow) is visible in the particles.

the uL4 TD (rpl4 $\Delta 63-87)$ vs. the TD being present but in a flexible state (nog1 $\mathrm{C}$ reil $\Delta C \operatorname{reh} 1 \Delta C)$. This milder effect in the nog1 mutant is also consistent with the observation that class N3, which contains the same aberrant rRNA conformations as class $\mathrm{R} 1$, makes up only $7 \%$ of the total particles obtained from the $n o g 1 \Delta C$ reil $\Delta C$ reh $1 \Delta C$ while class $\mathrm{R} 1$ makes up $27 \%$ of rpl4 $\Delta 63-87$ mutant particles (Supplementary Figs. 2b and $7 \mathrm{~b}$ ). When flexible, the TD of uL4 might still stabilize H74 enough to maintain growth at $30^{\circ} \mathrm{C}$ in rich media. However, the potential for this rRNA to become trapped in unproductive rRNA conformations might increase at lower temperatures and result in cold sensitivity ${ }^{36}$. Alternatively, or in addition, the lack of eL39 in the NPET of rpl4 $63-87$ mutant particles could help explain the difference between the rpl4 and nog1 mutants. While eL39 is not essential for growth, rpl39s mutants do exhibit growth defects ${ }^{37}$. Although it is unclear how eL39 is loaded into pre-60S subunits and why exactly deletion of the UL4 TD prevents this, eL39 may fine-tune NPET assembly.

Our results also suggest that Nog1 plays the most important role of the three tunnel-occupying AFs Nog1, Rei1, and Reh1 during ribosome assembly. Analogous C-terminal truncation of Reil has minimal effects on cell growth at all temperatures, while reh1 $\Delta$ cells 


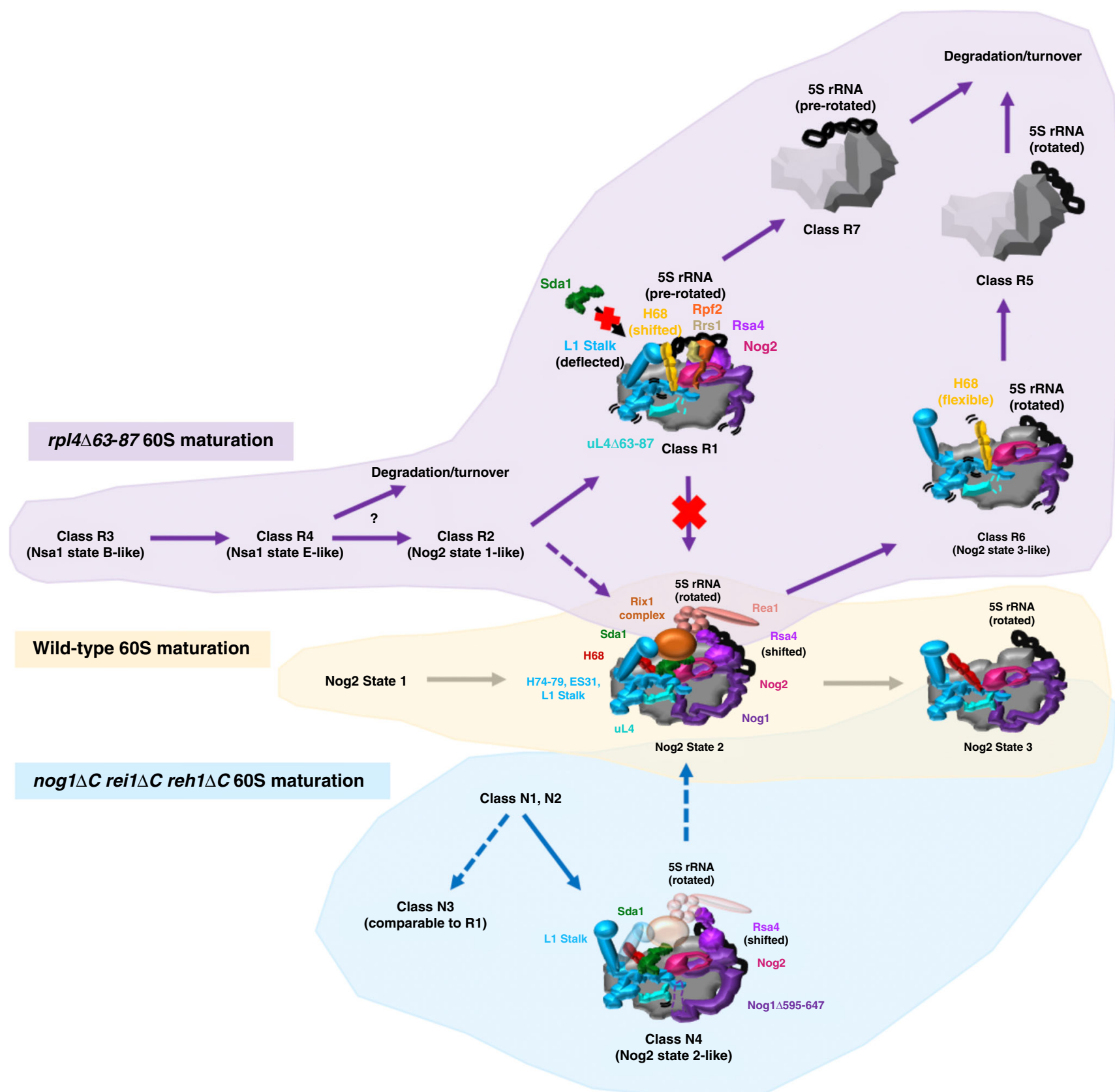

Fig. 8 NPET maturation in the 60 S subunit assembly hierarchy. In the wild-type 605 subunit assembly pathway (middle, tan), Nog2 state 1 progresses to Nog 2 state 2 particles, which have bound AFs Sda1 (dark green), the Rix 1 complex (orange), and Rea1 (coral). The L1 stalk has closed, and the 5S rRNA has rotated $\sim 180^{\circ}$. Rsa4 (light purple) has also shifted to face Rea1. These wild-type particles progress to Nog2 state three particles, which have released multiple AFs and are ready to exchange Nog2 for Nmd3 and be exported from the nucleoplasm to the cytoplasm. In the rp/4 $663-87$ mutant (top, purple) classes R3 and R4 are the earliest chronological particles to be observed. Some fraction presumably progresses to class R2. Class R2 particles are wildtype-like and likely progress to class R1 particles, which display a shifted rRNA helix H68 (gold) that prevents binding of Sda1 and progression to Nog2 state 2. These class R2 particles are likely precursors to class R7 on a pathway to turnover. Alternatively, some fraction of class R2 particles may progress past Nog2 state 2 to class R6, which displays aberrant conformations of multiple rRNA helices. Consequently, class R6 particles are likely precursors to class R5 on a pathway to turnover. In the nog $1 \Delta C$ rei1 $\Delta C$ reh1 $\Delta C$ mutant, wild-type-like classes N1 and N2 (bottom, teal) progress to either N3, which resembles class R1, or class N4. Class N4 primarily displays density for the L1 stalk in an open position and Sda1 is bound, but weak densities (transparent colors) can be seen for the Rix1 complex, Rea1, and the L1 stalk in a closed position. Some fraction of class N4 particles likely progresses to Nog2 state 3 . Two curved black lines represent flexibility in protein or rRNA structure. Solid vs. dotted arrows represent major and minor pathways, respectively.

have no obvious growth defect ${ }^{22,29}$. Nevertheless, our triple truncation mutant, $\operatorname{nog} 1 \Delta C \operatorname{rei} 1 \Delta C \operatorname{reh} 1 \Delta C$, displayed a slightly slower growth rate at $30^{\circ} \mathrm{C}$ than the nog $1 \Delta 595-647$ single mutant. This leaves the possibility that the NPET-occupying amino acids in the CTDs of either Reil or Reh1 perform a still unknown function.
Class $\mathrm{N} 4$ particles from the nog $1 \Delta C$ reil $\Delta C$ reh $1 \Delta C$ mutant resemble Nog2 state 2 particles but lack density for Real and display weak density for the Rixl complex (Fig. 7c). The only other deviation from wild-type Nog2 state 2 in class N4 is that the L1 stalk adopts an open conformation as opposed to a 
closed one. While this particle makes up a minor fraction of total particles $(5.6 \%)$, it is possible that failure to close the L1 stalk may explain why the Rix 1 complex and Real are not stably associated with pre-60S subunits. In this scenario, in wild-type cells, both Sda1 binding and closure of the L1 stalk may promote recruitment of the Rixl complex and Rea1. The flexible uL4 TD in the $\operatorname{nog} 1 \Delta C$ reil $\Delta C$ reh $1 \Delta C$ mutant may subtly perturb the conformation of $\mathrm{H} 74$, which could be propagated to $\mathrm{H} 75$ and then to H76 in the L1 stalk. These perturbations might affect the closing of the L1 stalk in ways that we do not yet fully understand.

A consistent defect observed across all cryo-EM classes from both mutants was the lack of the nonessential AF and export adaptor, Arx1 (Figs. 2d and 5c). Portions of the Nog1 CTD that lie just outside the NPET interact with Arx $1^{17}$. We reasoned that the flexibility or absence of the Nog1 CTD in our mutants may prevent stable assembly of Arx1. Normally, Reil is necessary for the removal of Arx1 from pre-60S subunits. When this fails to occur, Arxl remains bound to preribosomes as they are exported to the cytoplasm. However, when Arx1 cannot assemble into preribosomes, it is found in the nucleoplasm ${ }^{38}$. Consistent with the assembly defect of Arxl, we observed that upon truncation or tagging of the Nog1 CTD in an reil $\Delta$ strain, Arxl-GFP was located in the nucleoplasm (Supplementary Fig. 10a, b). These results indicate that the Nog1 CTD recruits Arxl to pre-60S subunits, contributing to the export competency of pre-60S subunits.

It is vital to the cell's survival that the constriction sites in the NPET are assembled properly, since these sites impact protein synthesis. In bacteria, deletion of the internal loop of uL4 results in a cold-sensitive growth defect and a failure to respond to the $\mathrm{cmlA}^{\mathrm{Crb}}$ pausing peptide during protein synthesis ${ }^{10}$. Mutations in the NPET have also been shown to confer resistance to the macrolide antibiotic, erythromycin ${ }^{14,39}$. Several previous results offered early clues that mechanisms may operate to couple NPET construction with other remodeling events during 60S subunit assembly $5,40,41$. For example, translating ribosomes that are stalled in response to small molecules, peptides, or antibiotics that bind to the NPET display a distorted PTC incapable of catalyzing peptide bond formation ${ }^{4}$. Furthermore, defects in bacterial ribosomes lacking a $5 \mathrm{~S}$ RNP can be partially rescued by the binding of a macrolide antibiotic, which binds to the NPET ${ }^{42}$. Our data and proposed model suggest that this communication between the NPET and other ribosomal domains can indeed occur during ribosome assembly, and that failure to do so can halt at least three stages of maturation.

In this study, we set out to gain a deeper understanding of how construction of a functional center such as the NPET could fit into the known hierarchy of $60 \mathrm{~S}$ subunit assembly. Since crucial features of the NPET lie deep within the core of the pre-60S subunit, cells need to be able to detect such subtle but impactful defects in NPET assembly. By coupling NPET construction with the stability of critical rRNA helices, cells might easily identify and terminate assembly of particles harboring a defective NPET. This study opens up the possibility that construction of other functional centers in the eukaryotic ribosome may be regulated in a similar fashion and lays the foundation for future study of mutant $60 \mathrm{~S}$ subunits using cryo-EM.

\section{Methods}

Construction of mutant plasmids. Plasmids containing each AF gene were obtained from the Yeast Genomic Tiling Collection (Open Biosystems), and used as templates in a multi-step PCR protocol for inserting unique restriction sites upstream and downstream of the gene ${ }^{43}$. PCR products were digested using restriction enzymes and ligated into pRS315 containing the LEU2 gene. Mutagenesis of ORFs was performed using the Quickchange Site-directed Mutagenesis Kit (Agilent Technologies). Correct mutations were confirmed by sequencing (Genewiz).
Generation of yeast strains. All genomic epitope tags, $G A L$-promoter fusions, knockouts, or C-terminal truncations were constructed according to standard methods ${ }^{44}$. The nog $1 \Delta C$ reil $\Delta C \operatorname{reh} 1 \Delta C$ triple truncation strain was made by truncating one assembly factor gene at a time. A strain conditional for expression of only $r p l 4 \Delta 63-87$ was constructed as follows. First the endogenous promoter of $R P L 4 A$ was replaced with the conditional GAL promoter, plus the HA-epitope inframe with the RPL4A ORF. Then, RPL4B was knocked out by replacement of the ORF with KanMX. This GAL-HA-RPL4A rpl4bD strain was transformed with a LEU2 plasmid pRS315 containing rpl4a $\Delta 63-87$. Arx1-GFP strains were a generous gift from Arlen Johnson (University of Texas at Austin). All strains and plasmids are available upon request. (Supplementary Tables 2 and 3).

Growth assays. Yeast strains were grown to an $\mathrm{OD}_{600}$ of $\sim 0.5$ in permissive conditions and then serially diluted out to $10^{-5}$-fold before pipetting $10-15 \mu \mathrm{L}$ of each dilution onto appropriate galactose- or glucose-containing solid media. Plates were checked daily after being incubated at 30 or $16^{\circ} \mathrm{C}$.

Sucrose density gradient analysis. Preribosomes, ribosomes, and polyribosomes were fractionated from $40 \mathrm{OD}_{254}$ units of yeast whole-cell extracts on 7-47\% (w/v) sucrose gradients ${ }^{30,45}$. Five milligrams of cycloheximide (Sigma) was added to $150 \mathrm{ml}$ of culture $20 \mathrm{~min}$ before harvesting the cells. Extracts were made using lysis buffer $\left(10 \mathrm{mM}\right.$ Tris- $\mathrm{HCl} \mathrm{pH} 7.5,0.1 \mathrm{M} \mathrm{NaCl}, 30 \mathrm{mM} \mathrm{MgCl}_{2}, 50 \mu \mathrm{g} / \mathrm{ml}$ cycloheximide, $200 \mu \mathrm{g} / \mathrm{ml}$ heparin, and $0.2 \%$ diethyl pyrocarbonate). Cells were vortexed eight times for $30 \mathrm{~s}$ with glass beads $(0.5 \mathrm{~mm}$ diameter, Biospec Products), kept on ice in between vortexing, and clarified by two consecutive centrifugations. Forty $\mathrm{OD}_{254}$ units of whole-cell extracts were layered on $7-47 \%(\mathrm{w} / \mathrm{v})$ sucrose gradients, and preribosomes, ribosomes, and polyribosomes were fractionated according to the manufacturer's protocol (Teledyne ISCO). The sucrose gradients were spun in an ultracentrifuge for $4 \mathrm{~h}$. A Foxy R1 density gradient fractionator was used to fractionate and analyze gradients with continuous monitoring at $\mathrm{OD}_{254}$.

Fluorescence microscopy. The intracellular location of preribosomes or AFs was determined as follows: $35 \mathrm{~mm}$ glass bottom microwell dishes (MaTek) were coated with concanavalin A (Sigma) and allowed to dry for $40 \mathrm{~min}$. Cells expressing GFPtagged uL23 or Arx1-GFP were grown to an $\mathrm{OD}_{600}$ of $0.3-0.5$ before plating $10 \mu \mathrm{L}$ onto the glass bottom of the MaTek dish and incubating at room temperature for $20 \mathrm{~min}$. Plates were then washed once with $1 \mathrm{~mL}$ of appropriate media before being overlaid with $2 \mathrm{~mL}$ of media and then imaged using a Ziess LSM 880 confocal microscope. Images were processed using Fiji ${ }^{46}$.

Analysis of pre-rRNA processing. Steady-state levels of pre-rRNAs were assayed using northern blot and primer extension anaysis ${ }^{47}$. Ten milliliter of cells were harvested, frozen, and RNA was extracted using phenol. Five microgram of RNA was used for primer extension reactions or loaded onto a formaldehyde/MOPS agarose gel for northern blotting. 32P $\gamma$-ATP radiolabeled oligonucleotide probes for specific pre-rRNAs were used in primer extension reactions and for hybridization in northern blots. For northern hybridization of small molecular weight RNAs $(7,6,5.8$, and 5S), RNA samples were mixed with an equal volume of sample buffer $(0.1 \times$ TBE buffer, $10 \mathrm{M}$ urea, $0.1 \%$ xylene cyanol, $0.1 \%$ bromophenol blue) and subjected to electrophoresis on a $5 \%$ acrylamide/7 $\mathrm{M}$ urea gel for $4 \mathrm{~h}$ at 120 $\mathrm{mA}$. Following electrophoresis, gels were electroblotted to a Nytran $\mathrm{N}$ membrane (GE Healthcare Life Sciences) using a Trans-Blot Plus Cell (Biorad), hybridized with an end-labeled oligonucleotide, washed, and exposed to X-ray film.

Affinity purification and analysis of preribosomes by silver staining, western blotting, and iTRAQ mass spectrometry. The protein composition profiles of affinity-purified preribosomes was analyzed by SDS-PAGE (4-20\% Tris-glycine and $4-12 \%$ Bis-Tris, Thermo Fisher Scientific) followed by silver staining ${ }^{33}$. Western blotting tested incorporation of specific proteins into preribosomes ${ }^{30}$. Preribosomes were purified by lysing frozen cells using acid-washed glass beads and TNM150 buffer consisting of $50 \mathrm{mM}$ Tris $\mathrm{HCl} \mathrm{pH} \mathrm{7.5,} 150 \mathrm{mM} \mathrm{NaCl}, 1.5 \mathrm{mM}$ $\mathrm{MgCl}_{2}, 0.1 \% \mathrm{NP}-40$ (Sigma-Aldrich), and $5 \mathrm{mM} \beta \mathrm{ME}$. Following lysis, lysates were incubated with IgG-conjugated Dynabeads while rocking for $1 \mathrm{~h}$ at $4{ }^{\circ} \mathrm{C}$. Dynabeads were washed with TNM150 buffer (for iTRAQ, this step excluded NP-40). Samples were eluted using TEV protease while rocking for $1 \mathrm{~h}$ at room temperature, then precipitated with 10\% TCA and washed with $100 \%$ cold acetone. Purified samples were sent to the Penn State Hershey Core Research Facilities for trypsin digestion and 4-plex labeling with iTRAQ reagents 114, 115, 116, 117 (Applied Biosystems).

Purification of preribosomes for cryo-EM. Preparation of Nog2-associated particles described here required $20 \mathrm{~L}$ of cells grown to an $\mathrm{OD}_{600}$ of $0.8-1.0$ in appropriate media. Frozen cell pellets were lysed using TAP lysis buffer consisting of $50 \mathrm{mM}$ Tris- $\mathrm{HCl} \mathrm{pH} 7.5,100 \mathrm{mM} \mathrm{NaCl}, 10 \mathrm{mM} \mathrm{MgCl}_{2}$, and $0.075 \% \mathrm{NP}-40$ (Sigma-Aldrich). Lysates were incubated with IgG-conjugated Dynabeads while rocking for $1 \mathrm{~h}$ at $4{ }^{\circ} \mathrm{C}$. Dynadeads were washed using TAP lysis buffer three times and then washed twice using TEV cleavage/resuspension buffer (TAP lysis buffer with NP-40 excluded). Samples were eluted using TEV protease while rocking for 
$1 \mathrm{~h}$ at room temperature. The eluate was then added to a $100 \mathrm{~K}$ centrifugal filter (Millipore), prewashed with TEV cleavage-resuspension buffer, and spun at $14,000 \times g$ for $5 \mathrm{~min}$ at $4{ }^{\circ} \mathrm{C}$. The flow through was discarded and the sample was recovered by inverting the filters and spinning again at $1000 \times g$ for $2 \mathrm{~min}$ before being stored at $-80^{\circ} \mathrm{C}$ until preparation for cryo-EM.

Cryo-EM data acquisition. Vitrified specimens were prepared by adding $4 \mu \mathrm{l}$ samples of nog $1 \Delta C$ reil $\Delta C$ reh $1 \Delta C$ or $r p l 4 \Delta 63-87$ particles at a concentration of $\sim 150 \mathrm{nM}$ to a glow-discharged holey carbon grid (Quantifoil R1.2/1.3) covered with a freshly made thin carbon film. Grids were blotted for $0.5 \mathrm{~s}$ and plungefrozen into liquid ethane using an FEI Vitrobot Mark IV $\left(4^{\circ} \mathrm{C}\right.$ and $100 \%$ humidity). The cryo-grids were initially screened at a nominal magnification of 92,000 $\times$ in an FEI Talos Arctica microscope, operated with an acceleration voltage of $200 \mathrm{kV}$. Good-quality grids were transferred to an FEI Titan Krios electron microscope that was operating at $300 \mathrm{kV}$. Images were recorded using a K2 Summit direct electron detector (Gatan) in counting mode at a nominal magnification of $\times 105,000$ (for the rpl4 $\Delta 63-87$ sample) and $\times 130,000$ (for the nog $1 \Delta C$ reil $\Delta C$ reh1 $\Delta C$ sample), respectively (pixel sizes on the object scale are 1.373 and $1.052 \AA$, respectively), with the defocus ranging from -1.0 to $-2.0 \mu \mathrm{m}$. Coma-free alignment was manually optimized and parallel illumination was verified before data collection. All micrographs with the K2 camera were collected semi-automatically by SerialEM ${ }^{48}$ under low-dose conditions. Each micrograph was dose-fractionated to 32 frames with a dose rate of $\sim 10.0$ counts per physical pixel per second for a total exposure time of $6.4 \mathrm{~s}$.

Cryo-EM data processing. Original image stacks were summed and corrected for drift and beam-induced motion at the micrograph level using the MotionCor2 program ${ }^{49}$. The SPIDER ${ }^{50}$ software was used for micrograph screening. The contrast transfer function parameters of each micrograph were estimated by Gctf (Zhang, K. 2016) ${ }^{51}$. All 2D and 3D classification and refinement were performed with RELION3.0 (Zivanov, J. et al. 2018) ${ }^{52}$. The local resolution map was estimated using ResMap ${ }^{53}$.

For the rpl4 $463-87$ mutant sample, a total of 5003 micrographs were collected and 883,649 particles were picked for a cascade $2 \mathrm{D}$ and $3 \mathrm{D}$ classification with a binning factor of two. About $57 \%$ of particles were removed during several rounds of $2 \mathrm{D}$ and $3 \mathrm{D}$ classification, and 382,748 particles were split into ten classes during the final round of 3D classification (Supplementary Fig. 2). After the final round of 3D classification, a total of 103,319 particles were applied for high-resolution refinement (without binning), resulting in a $3.12 \AA$ map (gold-standard FSC 0.143 criteria) (Supplementary Fig. 2c). A total of 47,025 particles were applied for highresolution refinement (without binning), resulting in a $3.22 \AA$ map (Supplementary Fig. 2d). In addition to these two states, class R3 is similar to the state B of Nsa1particles ${ }^{16}$ and class R4 is similar to the state E of Nsal-particles but with already assembled $5 \mathrm{~S}$ RNP (in the premature rotated state). Classes R5-R7 also have well resolved structural features; but they lack densities for major domains of rRNA, suggesting that they are likely turnover products (Supplementary Fig. 3a-e).

For the $\operatorname{nog} 1 \Delta C$ rei $1 \Delta C$ reh $1 \Delta C$ mutant sample, a total of 9115 micrographs were collected and 1,009,266 particles were picked for cascade 2D and 3D classification with a binning factor of four. About $67 \%$ of particles were removed during three rounds of 2D classification, and 332,827 particles were split into ten classes during the first round of 3D classification, with a map of the premature $60 \mathrm{~S}$ ribosomal subunit (EMD-6615) (low-pass filtered to $60 \AA$ ) as the initial model. Based on the map features (the presence of ITS2 and rotation of the 5S RNP), classes were combined into two groups and were subjected to a second round of 3D classification. The first group, with the $5 \mathrm{~S}$ RNP in premature state, was classified into ten classes. A majority of them (62.8\% particles, class N1) represent a state very similar to the state 1 of wild-type Nog2-particles ${ }^{17}$. A total of 89,239 particles were applied for high-resolution refinement (without binning), resulting in a $3.00 \AA$ map (Supplementary Fig. 7c). The second group, with the 5S RNP in mature-like position, containing 169,432 particles, was also subjected to a second round of $3 \mathrm{D}$ classification into ten classes. Around $62.6 \%$ of these particles are mature or mature-like $60 \mathrm{~S}$ subunits.

Model building and refinement. An atomic model of wild-type Nog2-TAP state 1 (PDB: $3 \mathrm{jct})^{17}$ was used as the initial template for modelling. The models of the rRNAs (25S, 5.8S, 5S, ITS2 RNA) were docked into the density map manually using UCSF Chimera ${ }^{54}$. For RP and AF modelling, structures of individual proteins were separately fitted into their density by rigid-body docking. After the initial fitting, the entire chains of rRNAs and proteins were manually checked and adjusted with $\mathrm{COOT}^{55}$. The atomic model of classes R1 and R2 of the rpl4 $463-87$ mutant particles were further refined against the density map first by real-space refinement (phenix.real_space_refine) ${ }^{56}$ in PHENIX ${ }^{57}$, with secondary structure restraints, geometry restraints and RNA-specific restraints applied. After refinement, alternating rounds of manual model adjustment using COOT and model refinement using PHENIX were applied. The atomic models were cross-validated according to standard procedures ${ }^{58,59}$. Specifically, the coordinates of the final model were randomly displaced by $0.2 \AA$ using the PDB tools of Phenix. The displaced model was refined against the Half1 map (produced from a half set of all particles during refinement by RELION). The refined model from
Half1 map was compared with the maps of Half1, Half2 in Fourier space to produce two FSC curves, FSC $_{\text {work }}$ (model versus Half1 map) and FSC $_{\text {free }}$ (model versus Half2 map), respectively (Supplementary Fig. 2c, d). Another FSC curve between the refined model from Half1 and the final density map (model versus full) from all particles was also produced. As indicated by these curves, the agreement between $\mathrm{FSC}_{\text {work }}$ and $\mathrm{FSC}_{\text {free }}$ (no large separation) indicated that the model was not overfitted. MolProbity ${ }^{60}$ (http://molprobity.biochem.duke.edu/) was used to evaluate the final model, and final statistics of the model are provided in Supplementary Table 1.

Statistics and reproducibility. The experiment shown in Fig. 2a was performed three times. The experiment shown in Fig. 4a was performed three times. The experiment shown in Fig. 4b was performed twice. The experiment shown in Fig. 5a was performed three times. Experiments shown in Fig. 7a, b were performed three times. The experiment shown in Supplementary Fig. la was performed three times. The experiment shown in Supplementary Fig. $1 \mathrm{~b}$ was performed twice. The experiments shown in Supplementary Fig. 6b, c were performed three times. The experiment shown in Supplementary Fig. 10a was performed twice

Reporting summary. Further information on research design is available in the Nature Research Reporting Summary linked to this article.

\section{Data availability}

The data that support this study are available from the corresponding authors upon reasonable request. The cryo-EM density maps of the R1, R2 classes of the rpl4 $\Delta 63-87$ mutant particles and the $\mathrm{N} 1, \mathrm{~N} 2, \mathrm{~N} 3, \mathrm{~N} 4$ classes of the $\operatorname{nog} 1 \Delta C$ reil $\Delta C$ reh1 $\Delta C$ mutant particles have been deposited in the Electron Microscopy Data Bank (EMDB) under accession numbers EMD-30170, EMD-30174, EMD-30172, EMD-30173, EMD-30175, and EMD-30176, respectively; and the atomic models of the R1 and R2 classes of the $r p l 4 \Delta 63-87$ mutant particles have been deposited in the Protein Data Bank (PDB) under accession numbers 7BT6 and 7BTB, respectively. Source data are provided with this paper.

Received: 28 January 2020; Accepted: 14 September 2020; Published online: 09 October 2020

\section{References}

1. Karbstein, K. Quality control mechanisms during ribosome maturation. Trends Cell Biol. 23, 242-250 (2013).

2. Peña, C., Hurt, E. \& Panse, V. G. Eukaryotic ribosome assembly, transport and quality control. Nat. Struct. Mol. Biol. 24, 689-699 (2017).

3. Mankin, A. S. Nascent peptide in the 'birth canal' of the ribosome. Trends Biochem. Sci. 31, 11-13 (2006).

4. Vázquez-Laslop, N. \& Mankin, A. S. How macrolide antibiotics Work. Trends Biochem. Sci. 43, 1-17 (2018).

5. Wilson, D. N., Arenz, S. \& Beckmann, R. Translation regulation via nascent polypeptide-mediated ribosome stalling. Curr. Opin. Struct. Biol. 37, 123-133 (2016)

6. Mankin, A. S. Picky nascent peptides do not talk to foreign ribosomes. Proc. Natl Acad. Sci. USA 108, 5931-5932 (2011).

7. Liutkute, M., Samatova, E. \& Rodnina, M. V. Cotranslational folding of proteins on the ribosome. Biomolecules 10, 1-5 (2020).

8. Jenner, L. et al. Crystal structure of the $80 \mathrm{~S}$ yeast ribosome. Curr. Opin. Struct. Biol. 22, 759-767 (2012)

9. Dao Duc, K., Batra, S. S., Bhattacharya, N., Cate, J. H. D. \& Song, Y. S. Differences in the path to exit the ribosome across the three domains of life. Nucleic Acids Res. 47, 4198-4210 (2019).

10. Lawrence, M. G. et al. The extended loops of ribosomal proteins uL4 and uL22 of Escherichia coli contribute to ribosome assembly and protein translation. Nucleic Acids Res. 44, 5798-5810 (2016).

11. Gamalinda, M. \& Woolford, J. L. Deletion of L4 domains reveals insights into the importance of ribosomal protein extensions in eukaryotic ribosome assembly. RNA 20, 1725-1731 (2014).

12. Stelter, P. et al. Coordinated ribosomal L4 protein assembly into the preribosome is regulated by its eukaryote-specific extension. Mol. Cell $\mathbf{5 8}$, 854-862 (2015)

13. Su, T. et al. The force-sensing peptide VemP employs extreme compaction and secondary structure formation to induce ribosomal stalling. elife https:// doi.org/10.7554/eLife.25642 (2017).

14. Wekselman, I. et al. The ribosomal protein uL22 modulates the shape of the protein exit tunnel article the ribosomal protein uL22 modulates the shape of the protein exit tunnel. Structure 25, 1-9 (2017).

15. Sanghai, Z. A. et al. Modular assembly of the nucleolar pre-60S ribosomal subunit. Nature 556, 126-129 (2018). 
16. Kater, L. et al. Visualizing the assembly pathway of nucleolar pre-60S ribosomes. Cell 171, 1599-1610 (2017).

17. Wu, S. et al. Diverse roles of assembly factors revealed by structures of late nuclear pre-60S ribosomes. Nature 534, 133-137 (2016).

18. Kappel, L. et al. Rlp24 activates the AAA-ATPase Drg1 to initiate cytoplasmic pre-60S maturation. J. Cell Biol. 199, 771-782 (2008).

19. Lo, K. Y. et al. Defining the pathway of cytoplasmic maturation of the $60 \mathrm{~S}$ ribosomal subunit. Mol. Cell 39, 196-208 (2010).

20. Greber, B. J., Boehringer, D., Montellese, C. \& Ban, N. Cryo-EM structures of Arx1 and maturation factors Reil and Jjj1 bound to the $60 \mathrm{~S}$ ribosomal subunit. Nat. Struct. Mol. Biol. 19, 1228-1233 (2012).

21. Ma, C. et al. Structural snapshot of cytoplasmic pre-60S ribosomal particles bound by Nmd3, Lsg1, Tif6 and Reh1. Nat. Struct. Mol. Biol. 24, 214-220 (2017).

22. Greber, B. J. et al. Insertion of the biogenesis factor Reil probes the ribosomal tunnel during 60S maturation. Cell 164, 91-102 (2016).

23. Barrio-Garcia, C. et al. Architecture of the Rix1-Real checkpoint machinery during pre-60S-ribosome remodeling. Nat. Struct. Mol. Biol. 23, 37-44 (2015).

24. Thoms, M. et al. Suppressor mutations in Rpf2-Rrs1 or Rpl5 bypass the Cgr1 function for pre-ribosomal 5S RNP-rotation. Nat. Commun. 9, 1-13 (2018).

25. Kater, L. et al. Construction of the central protuberance and L1 stalk during 60S subunit biogenesis. Mol. Cell 79, 1-14 (2020).

26. Zhou, Y., Musalgaonkar, S., Johnson, A. W. \& Taylor, D. Tightly-orchestrated rearrangements govern catalytic center assembly of the ribosome. Nat. Commun. 10, 1-34 (2018).

27. Tutuncuoglu, B., Jakovljevic, J., Wu, S., Gao, N. \& Woolford, J. L. The Nterminal extension of yeast ribosomal protein L8 is involved in two major remodeling events during late nuclear stages of $60 \mathrm{~S}$ ribosomal subunit assembly. RNA 22, 1386-1399 (2016).

28. Klinge, S. \& Woolford, J. L. Ribosome assembly coming into focus. Nat. Rev. Mol. Cell Biol. 20, 116-131 (2019).

29. Parnell, K. M. \& Bass, B. L. Functional redundancy of yeast proteins Reh1 and Rei1 in cytoplasmic 60S subunit maturation. Mol. Cell. Biol. 29, 4014-4023 (2009).

30. Biedka, S. et al. Hierarchical recruitment of ribosomal proteins and assembly factors remodels nucleolar pre-60S ribosomes. J. Cell Biol. 217, 2503-2518 (2018).

31. Sarkar, A. et al. Preribosomes escaping from the nucleus are caught during translation by cytoplasmic quality control. Nat. Struct. Mol. Biol. 24, 1107-1115 (2017).

32. Talkish, J. et al. Disruption of ribosome assembly in yeast blocks cotranscriptional pre-rRNA processing and affects the global hierarchy of ribosome biogenesis. RNA 22, 852-866 (2016).

33. Sahasranaman, A. et al. Assembly of Saccharomyces cerevisiae 60S ribosomal subunits: role of factors required for $27 \mathrm{~S}$ pre-rRNA processing. EMBO J. 30, 4020-4032 (2011).

34. Davis, J. H. et al. Modular assembly of the bacterial large ribosomal article modular assembly of the bacterial large ribosomal subunit. Cell 167, 1610-1622 (2016).

35. Mulder, A. et al. Visualizing ribosome biogenesis: parallel assembly pathways for the 30S subunit. Science 673, 673-677 (2011).

36. Chursov, A., Kopetzky, S. J., Bocharov, G., Frishman, D. \& Shneider, A. RNAtips: analysis of temperature-induced changes of RNA secondary structure. Nucleic Acids Res. 41, 486-491 (2013).

37. Steffen, K. K. et al. Ribosome deficiency protects against ER stress in Saccharomyces cerevisiae. Genetics 191, 107-118 (2012).

38. Hung, N.-J. \& Johnson, A. W. Nuclear recycling of the pre-60S ribosomal subunit-associated factor Arx1 depends on Reil in Saccharomyces cerevisiae. Mol. Cell. Biol. 26, 3718-3727 (2006).

39. Halfon, Y. et al. Exit tunnel modulation as resistance mechanism of S. aureus erythromycin resistant mutant. Sci. Rep. 9, 1-8 (2019).

40. Wu, C. et al. Arginine changes the conformation of the arginine attenuator peptide relative to the ribosome tunnel. J. Mol. Biol. 416, 518-533 (2012).

41. Gagnon, M. G. et al. Structures of proline-rich peptides bound to the ribosome reveal a common mechanism of protein synthesis inhibition. Nucleic Acids Res. 44, 2439-2450 (2016).

42. Khaitovich, P., Mankin, A. S. \& Ave, S. A. Effect of antibiotics on large ribosomal subunit assembly reveals possible function of $5 \mathrm{~S}$ rRNA. J. Mol. Biol. 291, 1025-1034 (1999).

43. Gamalinda, M. et al. A hierarchical model for assembly of eukaryotic $60 \mathrm{~S}$ ribosomal subunit domains. Genes Dev. 28, 198-210 (2014).

44. Longtine, M. S. et al. Additional modules for versatile and economical PCRbased gene deletion and modification in Saccharomyces cerevisiae. Yeast 14, 953-961 (1998).

45. Deshmukh, M., Tsay, Y., Paulovich, A. G. \& Woolford, J. L. Yeast ribosomal protein $\mathrm{Li}$ is required for the stability of newly synthesized $5 \mathrm{~S}$ rRNA and the assembly of 60S ribosomal subunits. Mol. Cell. Biol. 13, 2835-2845 (1993).
46. Schindelin, J. et al. Fiji: an open-source platform for biological-image analysis Nat. Methods 9, 676-682 (2012).

47. Jakovljevic, J. et al. Ribosomal proteins L7 and L8 function in concert with six $\mathrm{A}_{3}$ assembly factors to propagate assembly of domains I and II of $25 \mathrm{~S}$ rRNA in yeast 60S ribosomal subunits. RNA 18, 1805-1822 (2012).

48. Mastronarde, D. N. Automated electron microscope tomography using robust prediction of specimen movements. J. Struct. Biol. 152, 36-51 (2005).

49. Zheng, S. Q. et al. MotionCor2: anisotropic correction of beam-induced motion for improved cryo-electron microscopy. Nat. Methods 14, 331-332 (2017).

50. Shaikh, T. R. et al. Spider image processing for single-particle reconstruction of biological macromolecules from electron micrographs. Nat. Protoc. 3, 1941-1974 (2008)

51. Zhang, K. Gctf: Real-time CTF determination and correction. J. Struct. Biol. 193, 1-12 (2016).

52. Scheres, S. H. W. RELION: Implementation of a Bayesian approach to cryoEM structure determination. J. Struct. Biol. 180, 519-530 (2012).

53. Kucukelbir, A., Sigworth, F. J. \& Tagare, H. D. Quantifying the local resolution of cryo-EM density maps. Nat. Methods 11, 63-65 (2014).

54. Pettersen, E. F. et al. UCSF chimera-a visualization system for exploratory research and analysis. J. Comput. Chem. 25, 1605-1612 (2004).

55. Emsley, P. \& Lohkamp, B. Features and development of Coot. Biol. Crystallogr. https://doi.org/10.1107/S0907444910007493 (2010).

56. Afonine, P. V., Ralf, W., Headd, J. J. \& Thomas, C. Towards automated crystallographic structure refinement with phenix.refine. Biol. Crystallogr. https://doi.org/10.1107/S0907444912001308 (2012).

57. Adams, P. D. et al. PHENIX: a comprehensive Python-based system for macromolecular structure solution. Biol. Crystallogr. https://doi.org/10.1107/ S0907444909052925 (2010).

58. Zhao, M. et al. Mechanistic insights into the recycling machine of the SNARE complex. Nature 518, 61-67 (2015).

59. Amunts, A., Brown, A., Bai, X., Llácer, J. L. \& Hussain, T. Structure of the yeast mitochondrial large ribosomal subunit. Science 343, 1485-1489 (2014).

60. Chen, V. B. et al. MolProbity: all-atom structure validation for macromolecular crystallography. Acta Crystallogr. Sect. D. 66, 12-21 (2010).

\section{Acknowledgements}

We thank Jelena Micic, Fiona Fitzgerald, and Sebastian Klinge for critical reading of this manuscript and helpful discussions. We thank Cosmin Saveanu and Micheline Fromont-Racine (Nog2 and Tif6), Vikram Panse (Bud20 and Nug1), Janine Maddock (Nog1), Lasse Lindahl (uL4) $)^{10}$, and Doug Kellogg (Sda1), for generous gifts of antibodies. We also thank the Core Facilities at the School of Life Sciences, Peking University for help with negative staining EM; the Cryo-EM Platform of Peking University for help with data collection; the High-performance Computing Platform of Peking University for help with computation; and the National Centre for Protein Sciences at Peking University for assistance with sample preparation. This work was supported by the National Institutes of Health grant (R01GM028301 to J.L.W., Jr.), the National Science Foundation of China (31725007 and 31630087 to N.G.), and the Qidong-SLS Innovation Fund (to N.G.).

\section{Author contributions}

D.W. and J.W. designed the study. D.W., M.G., and A.L. constructed strains and plasmids, and D.W. and M.G. assayed effects of mutations on ribosome assembly. Y.L. collected the cryo-EM data and Y.L. and N.G. processed the data. Y.L built the atomic models, and together with D.W., N.G., and J.W., analyzed the structures. D.W., Y.L., N.G., and J.W. interpreted results and wrote the paper.

\section{Competing interests}

The authors declare no competing interests.

\section{Additional information}

Supplementary information is available for this paper at https://doi.org/10.1038/s41467 020-18878-8.

Correspondence and requests for materials should be addressed to N.G. or J.L.W.Jr.

Peer review information Nature Communications thanks the anonymous reviewers for their contribution to the peer review of this work. Peer reviewer reports are available.

Reprints and permission information is available at http://www.nature.com/reprints

Publisher's note Springer Nature remains neutral with regard to jurisdictional claims in published maps and institutional affiliations. 
(c) (i) Open Access This article is licensed under a Creative Commons Attribution 4.0 International License, which permits use, sharing, adaptation, distribution and reproduction in any medium or format, as long as you give appropriate credit to the original author(s) and the source, provide a link to the Creative Commons license, and indicate if changes were made. The images or other third party material in this article are included in the article's Creative Commons license, unless indicated otherwise in a credit line to the material. If material is not included in the article's Creative Commons license and your intended use is not permitted by statutory regulation or exceeds the permitted use, you will need to obtain permission directly from the copyright holder. To view a copy of this license, visit http://creativecommons.org/ licenses/by/4.0/.

(C) The Author(s) 2020 